\title{
Asymmetric Synthesis of a 5,6,7,8-Tetrahydro-1,6-
}

\section{naphthyridine Scaffold Leading to Potent Retinoid-Related}

\section{Orphan Receptor $\gamma \mathrm{t}$ Inverse Agonist TAK-828F}

Ryoji Tsuruoka,* Naoki Yoshikawa, * Takahiro Konishi and Mitsuhisa Yamano Process Chemistry, Pharmaceutical Sciences, Takeda Pharmaceutical Company Limited, 17-85 Jusohonmachi 2-Chome, Yodogawa-ku, Osaka 532-8686, Japan 
Table S1-1. Heck reaction: High throughput screening_1.

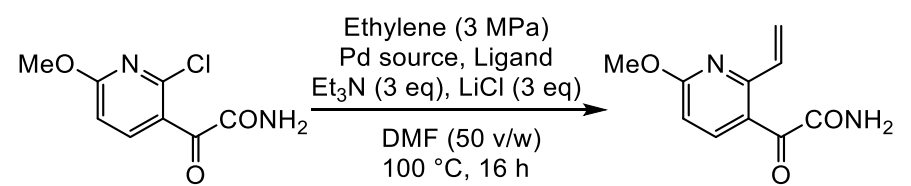

23

\begin{tabular}{|c|c|c|c|c|c|c|c|}
\hline \multirow{2}{*}{ Entry } & \multicolumn{2}{|l|}{ Pd source } & Ligand & \multicolumn{4}{|c|}{ HPLC area\% (220 nm) } \\
\hline & name & $\mathrm{mol} \%$ & name & $\mathrm{mol} \%$ & 23 & 19 & Others $^{a}$ \\
\hline 1 & $\mathrm{Pd}(\mathrm{OAc})_{2}$ & 20 & $\left(2,4,6-\mathrm{Me}_{3} \mathrm{Ph}\right)_{3} \mathrm{P}$ & 40 & 33.0 & 45.9 & 21.1 \\
\hline 2 & $\mathrm{Pd}(\mathrm{OAc})_{2}$ & 20 & $(\mathrm{o}-\mathrm{MeOPh})_{3} \mathrm{P}$ & 40 & 0.8 & 58.5 & 40.7 \\
\hline 3 & $\mathrm{Pd}(\mathrm{OAc})_{2}$ & 20 & $(p-\mathrm{MeOPh})_{3} \mathrm{P}$ & 40 & 18.0 & 15.1 & 66.9 \\
\hline 4 & $\mathrm{Pd}(\mathrm{OAc})_{2}$ & 20 & $(\mathrm{o}-\mathrm{Tol})_{3} \mathrm{P}$ & 40 & 42.5 & 40.4 & 17.1 \\
\hline 5 & $\mathrm{Pd}(\mathrm{OAc})_{2}$ & 20 & $(m-\mathrm{Tol})_{3} \mathrm{P}$ & 40 & 30.3 & 41.3 & 28.4 \\
\hline 6 & $\mathrm{Pd}(\mathrm{OAc})_{2}$ & 20 & $(p-\mathrm{Tol})_{3} \mathrm{P}$ & 40 & 23.9 & 49.2 & 26.9 \\
\hline 7 & $\mathrm{Pd}(\mathrm{OAc})_{2}$ & 20 & $(p-\mathrm{ClPh})_{3} \mathrm{P}$ & 40 & 28.3 & 35.5 & 36.2 \\
\hline 8 & $\mathrm{Pd}(\mathrm{OAc})_{2}$ & 20 & $\left(p-\mathrm{CF}_{3} \mathrm{Ph}\right)_{3} \mathrm{P}$ & 40 & 55.5 & 36.7 & 7.8 \\
\hline 9 & $\mathrm{Pd}(\mathrm{OAc})_{2}$ & 20 & $r a c-B I N A P$ & 20 & 33.2 & 3.1 & 63.7 \\
\hline 10 & $\mathrm{Pd}(\mathrm{OAc})_{2}$ & 20 & Xantphos & 20 & 12.3 & 70.7 & 17.0 \\
\hline 11 & $\mathrm{Pd}(\mathrm{OAc})_{2}$ & 20 & S-phos & 40 & 8.4 & 30.9 & 60.7 \\
\hline 12 & $\mathrm{Pd}(\mathrm{OAc})_{2}$ & 20 & X-phos & 40 & 86.8 & 7.8 & 5.4 \\
\hline 13 & $\mathrm{Pd}(\mathrm{OAc})_{2}$ & 20 & ${ }^{t} \mathrm{Bu}_{3} \mathrm{P} \cdot \mathrm{HBF}_{4}$ & 40 & 30.9 & 58.5 & 10.6 \\
\hline 14 & $\mathrm{PdCl}_{2}$ & 20 & $(\mathrm{o}-\mathrm{MeOPh})_{3} \mathrm{P}$ & 40 & N.D. & 66.9 & 33.1 \\
\hline 15 & $\mathrm{PdCl}_{2}$ & 20 & $(\mathrm{o}-\mathrm{Tol})_{3} \mathrm{P}$ & 40 & 29.6 & 51.0 & 19.4 \\
\hline 16 & $\mathrm{PdCl}_{2}$ & 20 & $(m-\mathrm{Tol})_{3} \mathrm{P}$ & 40 & 39.3 & 31.1 & 29.6 \\
\hline 17 & $\mathrm{PdCl}_{2}$ & 20 & $(p-\mathrm{Tol})_{3} \mathrm{P}$ & 40 & 8.6 & 62.1 & 29.3 \\
\hline 18 & $\mathrm{PdCl}_{2}$ & 20 & $(p-\mathrm{CIPh})_{3} \mathrm{P}$ & 40 & 26.0 & 36.4 & 37.6 \\
\hline 19 & $\left(\mathrm{Ph}_{3} \mathrm{P}\right)_{4} \mathrm{Pd}$ & 10 & - & & 37.2 & 4.9 & 57.9 \\
\hline 20 & {$\left[(0-\mathrm{TolP})_{3} \mathrm{P}\right]_{2} \mathrm{PdCl} 2$} & 10 & - & & 58.5 & 20.3 & 21.2 \\
\hline 21 & $\mathrm{Pd}(\mathrm{dppf}) \mathrm{Cl}_{2} \cdot \mathrm{CH}_{2} \mathrm{Cl}_{2}$ & 10 & - & & 69.6 & 13.8 & 16.6 \\
\hline 22 & $\mathrm{Pd}(\mathrm{dppe}) \mathrm{Cl}_{2}$ & 10 & - & & 63.9 & N.D. & 36.1 \\
\hline 23 & $\mathrm{Pd}(\mathrm{dppb}) \mathrm{Cl}_{2}$ & 10 & - & & 73.1 & 1.5 & 25.4 \\
\hline 24 & $\left({ }^{\mathrm{t}} \mathrm{Bu}{ }_{3} \mathrm{P}\right)_{2} \mathrm{Pd}$ & 10 & - & & 58.6 & 0.9 & 40.5 \\
\hline
\end{tabular}

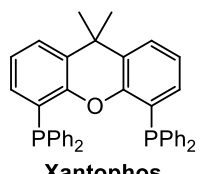

Xantophos
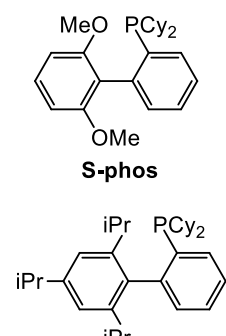

X-phos

ancluding the peak area of ligands. 
Table S1-2. Heck reaction: High throughput screening_2.<smiles>C=Cc1nc(OC)ccc1C(=O)C(N)=O</smiles>

23

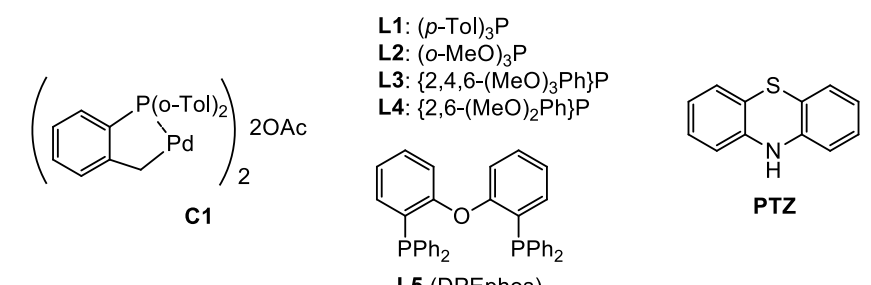

\begin{tabular}{|c|c|c|c|c|c|c|c|c|c|}
\hline \multirow{2}{*}{$\begin{array}{c}\text { Entr } \\
\mathrm{y}\end{array}$} & \multirow[b]{2}{*}{ Pd source (mol\%) } & \multirow{2}{*}{$\begin{array}{l}\text { Ligand } \\
\text { (mol\%) }\end{array}$} & \multirow{2}{*}{$\begin{array}{l}\text { DMF } \\
(v / w)\end{array}$} & \multirow[b]{2}{*}{ Base } & \multicolumn{2}{|c|}{ Additive(s) } & \multicolumn{3}{|c|}{ HPLC area\% $(220 \mathrm{~nm})$} \\
\hline & & & & & $\begin{array}{l}\mathrm{LiCl} \\
(\mathrm{eq})\end{array}$ & $\begin{array}{c}\text { PTZ } \\
(w / w \%)\end{array}$ & 23 & 19 & Others \\
\hline 1 & $\mathrm{PdCl}_{2}(20)$ & L1 (40) & 50 & $\mathrm{Et}_{3} \mathrm{~N}$ & 3 & - & 8.6 & 77.8 & 13.6 \\
\hline 2 & $\mathrm{PdCl}_{2}(10)$ & L1 (20) & 20 & $\mathrm{Et}_{3} \mathrm{~N}$ & 3 & - & 60.6 & 16.3 & 23.1 \\
\hline 3 & $\mathrm{PdCl}_{2}(20)$ & L1 (40) & 20 & $\mathrm{Et}_{3} \mathrm{~N}$ & 3 & - & 0.5 & 50.6 & 48.9 \\
\hline 4 & $\mathrm{PdCl}_{2}(20)$ & L1 (40) & 20 & $\mathrm{Et}_{3} \mathrm{~N}$ & 3 & 10 & 20.2 & 53.6 & 26.2 \\
\hline 5 & $\mathrm{PdCl}_{2}(20)$ & L1 (40) & 20 & $\mathrm{Et}_{3} \mathrm{~N}$ & 3 & $10^{a}$ & 25.8 & 45.9 & 28.3 \\
\hline 6 & $\mathrm{PdCl}_{2}(20)$ & L1 (40) & 20 & $\mathrm{Et}_{3} \mathrm{~N}$ & - & 10 & 0.5 & 79.9 & 19.6 \\
\hline 7 & $\mathrm{PdCl}_{2}(20)$ & L1 (40) & 20 & $\mathrm{Et}_{3} \mathrm{~N}$ & - & - & 0.4 & 76.6 & 23.0 \\
\hline 8 & $\mathrm{PdCl}_{2}(20)$ & L1 (40) & 10 & $\mathrm{Et}_{3} \mathrm{~N}$ & 3 & 10 & 43.3 & 7.5 & 49.2 \\
\hline 9 & $\mathrm{PdCl}_{2}(20)$ & L1 (40) & 20 & AcOK & 3 & 10 & 0.4 & 66.1 & 33.5 \\
\hline 10 & $\mathrm{PdCl}_{2}(20)$ & L1 (40) & 20 & $\mathrm{~K}_{2} \mathrm{CO}_{3}$ & 3 & 10 & 0.4 & 66.8 & 32.8 \\
\hline 11 & $\mathrm{PdCl}_{2}(20)$ & L1 (40) & 20 & $\mathrm{~K}_{3} \mathrm{PO}_{4}$ & 3 & 10 & 0.3 & 81.8 & 17.9 \\
\hline 12 & $\mathrm{PdCl}_{2}(20)$ & L1 (40) & 20 & ${ }^{i} \mathrm{Pr}_{2} \mathrm{EtN}$ & 3 & 10 & 5.5 & 65.3 & 29.2 \\
\hline 13 & $\mathrm{PdCl}_{2}(20)$ & L1 (40) & 20 & $\mathrm{Cs}_{2} \mathrm{CO}_{3}$ & 3 & 10 & 16.0 & 64.6 & 19.4 \\
\hline 14 & $\mathrm{PdCl}_{2}(20)$ & L1 (60) & 20 & $\mathrm{Et}_{3} \mathrm{~N}$ & 3 & 10 & 67.5 & 8.3 & 24.2 \\
\hline 15 & $\mathrm{PdCl}_{2}(20)$ & L2 (40) & 20 & $\mathrm{Et}_{3} \mathrm{~N}$ & 3 & 10 & 43.6 & 9.2 & 47.2 \\
\hline 16 & $\mathrm{PdCl}_{2}(20)$ & L2 (40) & 20 & $\mathrm{Et}_{3} \mathrm{~N}$ & 3 & - & 12.1 & 20.6 & 67.3 \\
\hline 17 & $\mathrm{PdCl}_{2}(20)$ & L2 (40) & 10 & $\mathrm{Et}_{3} \mathrm{~N}$ & 3 & 10 & 43.9 & 2.6 & 53.5 \\
\hline 18 & $\mathrm{PdCl}_{2}(20)$ & L3 (40) & 20 & $\mathrm{Et}_{3} \mathrm{~N}$ & 3 & 10 & 26.0 & 1.1 & 72.9 \\
\hline 19 & $\mathrm{PdCl}_{2}(20)$ & L4 (40) & 20 & $\mathrm{Et}_{3} \mathrm{~N}$ & 3 & 10 & 43.9 & 2.6 & 53.5 \\
\hline 20 & $\mathrm{PdCl}_{2}(20)$ & L5 (20) & 20 & $\mathrm{Et}_{3} \mathrm{~N}$ & 3 & 10 & 0.9 & 61.1 & 38.0 \\
\hline 21 & $\mathrm{Pd}(\mathrm{acac})_{2}(20)$ & L1 (40) & 20 & $\mathrm{Et}_{3} \mathrm{~N}$ & 3 & 10 & 32.6 & 28.9 & 38.5 \\
\hline 22 & $\mathrm{Pd}_{2}(\mathrm{dba})_{3}(10)$ & L1 (40) & 20 & $\mathrm{Et}_{3} \mathrm{~N}$ & 3 & 10 & 37.4 & 10.1 & 52.5 \\
\hline 23 & $\mathrm{Pd}\left(\mathrm{CF}_{3} \mathrm{COO}\right)_{2}(20)$ & L1 (40) & 20 & $\mathrm{Et}_{3} \mathrm{~N}$ & 3 & 10 & 38.7 & 34.8 & 26.5 \\
\hline 24 & C1 (20) & - & 20 & $\mathrm{Et}_{3} \mathrm{~N}$ & 3 & 10 & 82.0 & 0.0 & 18.0 \\
\hline
\end{tabular}

a. Dibutylhydroxytoluene (BHT) was used instead of PTZ 
Table S1-3. Heck reaction: High throughput screening_3.

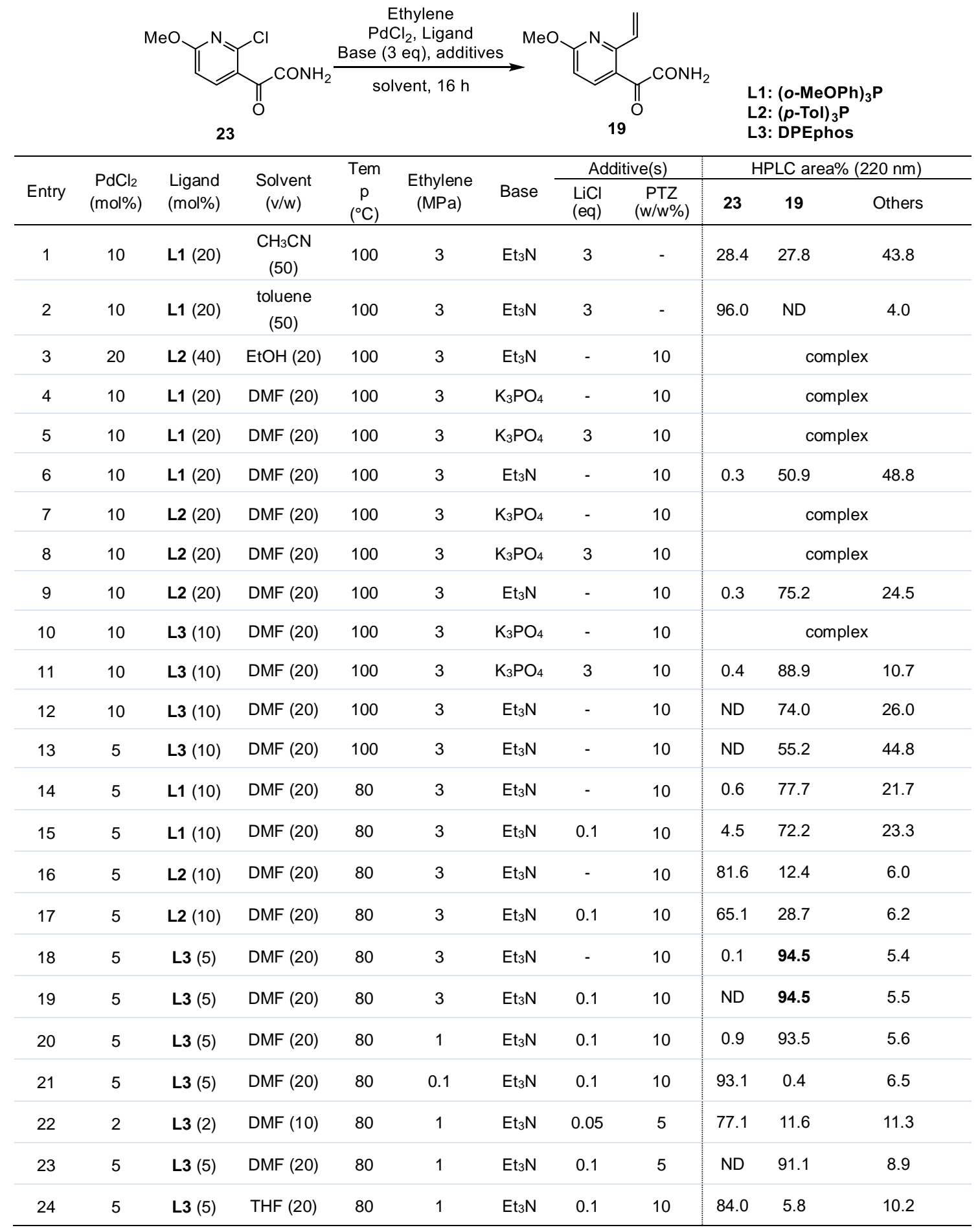


Table S2 Screening of the enantioselective reduction of $\mathbf{1 7}$<smiles>COc1ccc2c(n1)CCNC2C(N)=O</smiles>

S2-1 Ru catalyzed hydrogenation $\left(\mathrm{H}_{2} 3 \mathrm{MPa}, \mathrm{MeOH}(\mathrm{s} / \mathrm{s}=25), 40^{\circ} \mathrm{C}, 21 \mathrm{~h}\right)$

\begin{tabular}{|c|c|c|c|c|c|}
\hline Entry & Ccatalyst & $\begin{array}{l}\text { additive } \\
\text { (10eq. vs cat) }\end{array}$ & conv. $\%$ & ee\% & config. \\
\hline 1 & $\operatorname{RuCl}_{2}\{(S)$-binap $\}\{(S, S)$-dpen $\}$ & 'BuOK & 25.9 & 100.0 & $R$ \\
\hline 2 & $\operatorname{RuCl}_{2}\{(S)$-binap $\}\{(S)$-daipen $\}$ & ${ }^{\mathrm{t}} \mathrm{BuOK}$ & 21.4 & 100.0 & $R$ \\
\hline 3 & {$\left[\operatorname{RuCl}_{2}\{(R)\right.$-xylbinap $\}\{(R, R)$-dpen $\left.\}\right]$} & 'BuOK & 35.5 & 96.9 & $R$ \\
\hline 4 & {$[\operatorname{Ru}\{(S)$-np-binap $\}](\mathrm{OAc})_{2}$} & - & 42.7 & 87.5 & $R$ \\
\hline 5 & $\begin{array}{l}\text { Chloro[(R)-(+)-5,5'-bis(diphenylphosphino)-4,4-bi-1,3-benzodioxole](P- } \\
\text { cymene)ruthenium(II) chloride }\end{array}$ & ${ }^{\mathrm{t}} \mathrm{BuOK}$ & 34.8 & 82.8 & $R$ \\
\hline 6 & 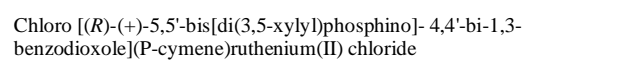 & tBuOK & 26.5 & 69.9 & $R$ \\
\hline 7 & $\mathrm{Ru}(\mathrm{OAc})_{2}\{(S)$-segphos $\}$ & & 18.0 & 64.8 & $S$ \\
\hline 8 & $\mathrm{Ru}(\mathrm{OAc})_{2}\{(S)$-binap $\}$ & 'BuOK & 4.7 & 56.2 & $R$ \\
\hline 9 & $\mathrm{Ru}(\mathrm{OAc})_{2}\left\{(S)-\mathrm{H}_{8}\right.$-binap $\}$ & tBuOK & 6.1 & 55.2 & $R$ \\
\hline 10 & {$[\mathrm{Ru}\{(S)$-pfp-binap $\}](\mathrm{OAc})_{2}$} & & 60.5 & 49.5 & $R$ \\
\hline 11 & $\mathrm{Ru}(\mathrm{OAc})_{2}[(S)$-binap $]$ & - & 22.8 & 46.2 & $R$ \\
\hline 12 & {$\left[\operatorname{RuCl}_{2}\{(R)\right.$-xylbinap $\}\{(R, R)$-dpen $\left.\}\right]$} & $\mathrm{KOH}$ & 11.7 & 44.3 & $S$ \\
\hline 13 & {$[\operatorname{Ru}\{(S)$-bop-binap $\}](\mathrm{OAc})_{2}$} & - & 23.5 & 35.2 & $R$ \\
\hline 14 & $\operatorname{RuCl}[(S, S)$-iBuSO 2 dpen $]($ p-cymene $)$ & ${ }^{\mathrm{t}} \mathrm{BuOK}$ & 29.9 & 29.2 & $S$ \\
\hline 15 & {$\left[\operatorname{RuCl}_{2}\{(S)\right.$-dadmpbinap $\}\{(S, S)$-dpen $\left.\}\right]$} & 'BuOK & 3.2 & 10.0 & $R$ \\
\hline 16 & {$\left[\operatorname{RuCl}_{2}\{(S)\right.$-xylbinap $\}\{(S)$-daipen $\left.\}\right]$} & tBuOK & 0.0 & 0.0 & - \\
\hline 17 & {$\left[\operatorname{RuCl}_{2}\{(S)\right.$-dadmpbinap $\}\{(S)$-daipen $\left.\}\right]$} & 'BuOK & 0.0 & 0.0 & - \\
\hline 18 & $\mathrm{RuCl}_{2}[(S)$-dtbm-binap $](\mathrm{OAc})_{2}$ & - & 0.0 & 0.0 & - \\
\hline 19 & $\mathrm{RuCl}_{2}[(S)$-dm-segphos][(S,S)-dpen] & 'BuOK & 0.0 & 0.0 & - \\
\hline 20 & $\mathrm{RuCl}_{2}[(S)$-dm-segphos $][(S)$-daipen $]$ & 'BuOK & 0.0 & 0.0 & - \\
\hline 21 & {$[\operatorname{RuBr} 2((S, S)$-xylskewphos $)((S, S)$-dpen $)]$} & 'BuOK & 0.0 & 0.0 & - \\
\hline 22 & $(R)-\mathrm{RuCl}[($ p-cymene $)(\mathrm{DTBM}$-segphos) $] \mathrm{Cl}$ & 'BuOK & 0.0 & 0.0 & - \\
\hline 23 & $(R)$-RUCY-XylBINAP & 'BuOK & 0.0 & 0.0 & - \\
\hline 24 & $\operatorname{RuCl}_{2}\{(R)-\operatorname{Pr}$ duphos $\}(\mathrm{dmf})_{\mathrm{n}}$ & & 0.0 & 0.0 & - \\
\hline
\end{tabular}


S2-2 Ir catalyzed hydrogenation ( $\operatorname{Ir}$ source : $[\operatorname{Ir}(\operatorname{cod}) \mathrm{Cl}]_{2}, \mathrm{H}_{2} 3 \mathrm{MPa}, 10 \mathrm{v} / \mathrm{v} \% \mathrm{AcOH} /$ Toluene $(\mathrm{S} / \mathrm{S}=35)$, Tetra n-butylammonium iodide(10eq. vs Ir), $40{ }^{\circ} \mathrm{C}, 21 \mathrm{~h}$ )

\begin{tabular}{|c|c|c|c|c|}
\hline Entry & ligand & conv. $\%$ & ee $\%$ & config. \\
\hline 1 & $(R)-(S)-(1-\mathrm{Naphtyl})_{2} \mathrm{PF}-\mathrm{P}^{\mathrm{t}} \mathrm{Bu}_{2}$ & 93.5 & 34.2 & $R$ \\
\hline 2 & $(R)-(S)-\mathrm{Bu}_{2} \mathrm{PF}-\mathrm{PPh}_{2}$ & 44.1 & 28.1 & $S$ \\
\hline 3 & $(R)-(S)-\mathrm{PPPhCHNMe} 2-\mathrm{F}-\mathrm{PP}$ & 91.7 & 18.9 & $S$ \\
\hline 4 & $(R)-(S)-\left(3,5-\mathrm{Me}_{2}-4-\mathrm{MeOPh}\right)_{2} \mathrm{PF}-\mathrm{P}^{\mathrm{t} B u} 2$ & 88.5 & 14.1 & $R$ \\
\hline 5 & $(R)-(S)-(4-\mathrm{MeO}-3,5-\mathrm{MePh})_{2} \mathrm{PF}-\mathrm{Pcy}{ }_{2}$ & 100.0 & 11.8 & $R$ \\
\hline 6 & $(R)-(S)-\mathrm{t} \mathrm{Bu}_{2} \mathrm{PF}-\mathrm{PoTol}_{2}$ & 78.3 & 11.6 & $R$ \\
\hline 7 & $(R)-(S)-(1-\mathrm{Naphtyl})_{2} \mathrm{PF}-\mathrm{PXy} \mathrm{l}_{2}$ & 98.6 & 11.5 & $S$ \\
\hline 8 & $(R)-(S)-\left(3,5-\mathrm{Me}_{2}-4-\mathrm{MeOPh}\right)_{2} \mathrm{PF}-\mathrm{Pxyl}_{2}$ & 93.7 & 10.4 & $S$ \\
\hline 9 & $(R)-(S)-\mathrm{PPF}-\mathrm{Pcy}_{2}$ & 100.0 & 8.4 & $R$ \\
\hline 10 & $(R)-(S)-2-\mathrm{Fur}_{2} \mathrm{PF}-\mathrm{Pxyl}_{2}$ & 100.0 & 7.9 & $R$ \\
\hline 11 & $(R)$-Ph-MeO-BIPHEP & 42.4 & 7.4 & $S$ \\
\hline 12 & $(R)-(S)-\left(3,5-\mathrm{CF}_{3} \mathrm{Ph}\right)_{2} \mathrm{PF}-\mathrm{Pxyl}_{2}$ & 100.0 & 6.9 & $S$ \\
\hline 13 & $(R)-(S)-\left(4-\mathrm{CF}_{3} \mathrm{Ph}\right)_{2}-\mathrm{P}^{t} \mathrm{Bu}_{2}$ & 88.2 & 6.9 & $S$ \\
\hline 14 & $(R)-(S)-\mathrm{Fur}_{2} \mathrm{PF}-\mathrm{P}(\mathrm{o}-\mathrm{Tol})_{2}$ & 100.0 & 6.6 & $R$ \\
\hline 15 & $(R)-(R)-\mathrm{PPPhFCHCH}_{3} \mathrm{P}\left(\left(3,5-\mathrm{CF}_{3}\right)_{2} \mathrm{Ph}\right)_{2}$ & 100.0 & 5.5 & $S$ \\
\hline 16 & $(R)-(S)-\mathrm{PPF}-\mathrm{P}^{\mathrm{t}} \mathrm{Bu}_{2}$ & 87.0 & 5.1 & $R$ \\
\hline 17 & $(R)-(S)-\mathrm{cy}_{2} \mathrm{PF}-\mathrm{PP}$ & 100.0 & 3.9 & $R$ \\
\hline 18 & $(R)-(S)-\left(3,5-\mathrm{CF}_{3} \mathrm{Ph}\right)_{2} \mathrm{PF}-\mathrm{Pcy} 2$ & 78.3 & 3.9 & $S$ \\
\hline 19 & $(R)-(S)-\mathrm{cy}_{2} \mathrm{PF}-\mathrm{Pcy}{ }_{2}$ & 100.0 & 3.0 & $S$ \\
\hline 20 & $(R)-(S)$-cy $2 \mathrm{PF}-\mathrm{PtBu}_{2}$ & 100.0 & 2.6 & $R$ \\
\hline 21 & $(R)-(S)-\mathrm{NMe}_{2}-\mathrm{PPh}_{2}-\mathrm{Mandyphos}$ & 100.0 & 2.5 & $S$ \\
\hline 22 & $(R)-(S)-\left(3,5-\mathrm{Me}_{2}-4-\mathrm{MeOPh}\right)_{2} \mathrm{PF}-\mathrm{PoTol}_{2}$ & 100.0 & 1.7 & $R$ \\
\hline 23 & $(R)-(S)-\mathrm{PPF}-\mathrm{Pxy}_{2}$ & 100.0 & 1.3 & $R$ \\
\hline 24 & $(R)-(S)-2-\mathrm{Fur}_{2} \mathrm{PF}-\mathrm{PtBu}_{2}$ & 89.4 & 1.1 & $R$ \\
\hline
\end{tabular}


S2-3 Rh catalyzed hydrogenation $\left(\mathrm{H}_{2} 3 \mathrm{MPa}, 40{ }^{\circ} \mathrm{C}, 21 \mathrm{~h}\right)$

\begin{tabular}{|c|c|c|c|c|}
\hline Entry & ligand, (metal source), solv. & conv. $\%$ & $\mathrm{ee} \%$ & config. \\
\hline 1 & (R)-Ph-MeO-BIPHEP+[Rh(cod)Cl $]_{2}, \mathrm{MeOH}, \mathrm{THF}$ & 29.6 & 87.3 & $R$ \\
\hline 2 & $(R)-(S)-\mathrm{Fur}_{2} \mathrm{PF}-\mathrm{P}(\mathrm{o}-\mathrm{Tol})_{2}+[\mathrm{Rh}(\mathrm{cod}) \mathrm{Cl}]_{2}, \mathrm{MeOH}, \mathrm{THF}$ & 9.2 & 86.0 & $R$ \\
\hline 3 & $(R)-(S)-\mathrm{PPF}-\mathrm{P}^{\mathrm{t}} \mathrm{Bu}_{2}+[\mathrm{Rh}(\mathrm{cod}) \mathrm{Cl}]_{2}, \mathrm{MeOH}, \mathrm{THF}$ & 82.3 & 85.5 & $R$ \\
\hline 4 & $(R)-(S)-\left(3,5-\mathrm{Me}_{2}-4-\mathrm{MeOPh}\right)_{2} \mathrm{PF}-\mathrm{P}^{t} \mathrm{Bu}_{2}+[\mathrm{Rh}(\mathrm{cod}) \mathrm{Cl}]_{2}, \mathrm{MeOH}, \mathrm{THF}$ & 88.8 & 85.3 & $R$ \\
\hline 5 & $(R)-(S)-\left(3,5-\mathrm{CF}_{3} \mathrm{Ph}\right)_{2} \mathrm{PF}-\mathrm{Pxyl}_{2}+[\mathrm{Rh}(\mathrm{cod}) \mathrm{Cl}]_{2}, \mathrm{MeOH}, \mathrm{THF}$ & 25.8 & 83.0 & $S$ \\
\hline 6 & $(R)-(S)-(1-\mathrm{Naphtyl})_{2} \mathrm{PF}-\mathrm{PXy} l_{2}+[\mathrm{Rh}(\mathrm{cod}) \mathrm{Cl}]_{2}, \mathrm{MeOH}, \mathrm{THF}$ & 10.6 & 66.3 & $S$ \\
\hline 7 & $(R)-(S)-2-\mathrm{Fur}_{2} \mathrm{PF}-\mathrm{P}^{\mathrm{t}} \mathrm{Bu}_{2}+[\mathrm{Rh}(\mathrm{cod}) \mathrm{Cl}]_{2}, \mathrm{MeOH}, \mathrm{THF}$ & 29.2 & 59.0 & $R$ \\
\hline 8 & $(R)-(S)-\mathrm{cy}_{2} \mathrm{PF}-\mathrm{P}^{\mathrm{t}} \mathrm{Bu}_{2}+[\mathrm{Rh}(\mathrm{cod}) \mathrm{Cl}]_{2}, \mathrm{MeOH}, \mathrm{THF}$ & 99.1 & 56.6 & $S$ \\
\hline 9 & $(R)-(S)-\left(4-\mathrm{CF}_{3} \mathrm{Ph}\right)_{2}-\mathrm{P}^{\mathrm{t}} \mathrm{Bu}_{2}+[\mathrm{Rh}(\mathrm{cod}) \mathrm{Cl}]_{2}, \mathrm{MeOH}, \mathrm{THF}$ & 64.3 & 51.5 & $R$ \\
\hline 10 & $(R)-(S)-\mathrm{t} \mathrm{Bu}_{2} \mathrm{PF}-\mathrm{PPh}_{2}+[\mathrm{Rh}(\mathrm{cod}) \mathrm{Cl}]_{2}, \mathrm{MeOH}, \mathrm{THF}$ & 22.1 & 50.6 & $R$ \\
\hline 11 & $(R)-(S)-\mathrm{NMe}_{2}-\mathrm{PPh}_{2}-\mathrm{Mandyphos}+[\mathrm{Rh}(\mathrm{cod}) \mathrm{Cl}]_{2}, \mathrm{MeOH}, \mathrm{THF}$ & 17.5 & 38.3 & $R$ \\
\hline 12 & $(R)-(S)-\mathrm{cy}_{2} \mathrm{PF}-\mathrm{Pcy}_{2}+[\mathrm{Rh}(\mathrm{cod}) \mathrm{Cl}]_{2}, \mathrm{MeOH}, \mathrm{THF}$ & 37.2 & 36.7 & $R$ \\
\hline 13 & {$[\mathrm{Rh}(\operatorname{cod})\{(R)-(S)-\mathrm{Josiphos}] \mathrm{OTf}, \mathrm{MeOH}$} & 95.3 & 36.2 & $S$ \\
\hline 14 & {$[\mathrm{Rh}(\operatorname{cod})\{(S)$-binap $\}] \mathrm{ClO}_{4}, \mathrm{MeOH}$} & 53.4 & 34.3 & $R$ \\
\hline 15 & $(R, R)-\mathrm{Me}-\mathrm{BPE}-\mathrm{Rh}, \mathrm{MeOH}$ & 99.5 & 34.0 & $R$ \\
\hline 16 & {$[\mathrm{Rh}(\operatorname{cod})\{(2 S, 4 S)-$ EtFerroTANE $\}] \mathrm{BF}_{4}, \mathrm{MeOH}$} & 56.3 & 32.6 & $R$ \\
\hline 17 & $(R, R)-\mathrm{Ph}-\mathrm{BPE}-\mathrm{Rh}, \mathrm{MeOH}, \mathrm{THF}, \mathrm{MeOH}$ & 99.1 & 31.7 & $S$ \\
\hline 18 & {$\left[\mathrm{Rh}(\operatorname{cod})\{(2 S, 4 S)-\mathrm{EtFerroTANE}\} \mathrm{BF}_{4}, \mathrm{IPA}\right.$} & 25.8 & 29.8 & $S$ \\
\hline 19 & $(R)-(S)-\mathrm{PPF}-\mathrm{Pxyl}_{2}+[\mathrm{Rh}(\mathrm{cod}) \mathrm{Cl}]_{2}, \mathrm{MeOH}, \mathrm{THF}$ & 21.5 & 29.3 & $S$ \\
\hline 20 & $(R)-(S)-\mathrm{PPF}-\mathrm{Pcy}_{2}+[\mathrm{Rh}(\mathrm{cod}) \mathrm{Cl}]_{2}, \mathrm{MeOH}, \mathrm{THF}$ & 23.7 & 28.5 & $S$ \\
\hline 21 & $(R, R)-\mathrm{Me}-\mathrm{BPE}-\mathrm{Rh}, \mathrm{IPA}$ & 93.8 & 28.0 & $S$ \\
\hline 22 & $(R)-(S)-(4-\mathrm{MeO}-3,5-\mathrm{MePh})_{2} \mathrm{PF}-\mathrm{Pcy}_{2}+[\mathrm{Rh}(\mathrm{cod}) \mathrm{Cl}]_{2}, \mathrm{MeOH}, \mathrm{THF}$ & 69.0 & 23.9 & $S$ \\
\hline 23 & $\operatorname{Rh}(\operatorname{cod})(S, S)$-ptbp-skewphos OTf,IPA & 92.1 & 23.3 & $R$ \\
\hline 24 & $(R)-(S)-(1-\mathrm{Naphtyl})_{2} \mathrm{PF}-\mathrm{PtBu}_{2}+[\mathrm{Rh}(\operatorname{cod}) \mathrm{Cl}]_{2}, \mathrm{MeOH}, \mathrm{THF}$ & 42.9 & 23.2 & $R$ \\
\hline 25 & $\begin{array}{l}R \text {-(tBu-methylphosphino-di-tBu-phosphinomethane)- } \\
\text { n4-(1,5-cod)Rh(I) } \mathrm{BF}_{4}, \mathrm{MeOH}\end{array}$ & 94.7 & 22.8 & $R$ \\
\hline 26 & $\mathrm{Rh}(\operatorname{cod})(S, S)$-ptbp-skewphos OTf,MeOH & 97.4 & 22.0 & $R$ \\
\hline 27 & $(R)-(S)-\mathrm{PPPhCHNMe}-\mathrm{F}-\mathrm{PP}+[\mathrm{Rh}(\mathrm{cod}) \mathrm{Cl}]_{2}, \mathrm{MeOH}, \mathrm{THF}$ & 24.4 & 21.7 & $R$ \\
\hline 28 & $(R, R)-\mathrm{Ph}-\mathrm{BPE}-\mathrm{Rh}, \mathrm{IPA}$ & 70.0 & 20.3 & $S$ \\
\hline 29 & $(R)-(S)-2-\mathrm{Fur}_{2} \mathrm{PF}-\mathrm{Pxyl}_{2}+[\mathrm{Rh}(\mathrm{cod}) \mathrm{Cl}]_{2}, \mathrm{MeOH}, \mathrm{THF}$ & 40.0 & 18.7 & $S$ \\
\hline 30 & {$[\mathrm{Rh}(\operatorname{cod})\{(2 S, 5 S)-\mathrm{EtDuphos}\}] \mathrm{OTf}, \mathrm{MeOH}$} & 74.4 & 18.5 & $S$ \\
\hline 31 & $\begin{array}{l}R \text {-(tBu-methylphosphino-di-tBu-phosphinomethane)- } \\
\text { n4-(1,5-cod)Rh(I) } \mathrm{BF}_{4}, \mathrm{IPA}\end{array}$ & 45.4 & 17.0 & $R$ \\
\hline 32 & $(R)-(S)-\mathrm{cy}_{2} \mathrm{PF}-\mathrm{PP}+[\mathrm{Rh}(\mathrm{cod}) \mathrm{Cl}]_{2}, \mathrm{MeOH}, \mathrm{THF}$ & 20.2 & 16.9 & $S$ \\
\hline
\end{tabular}




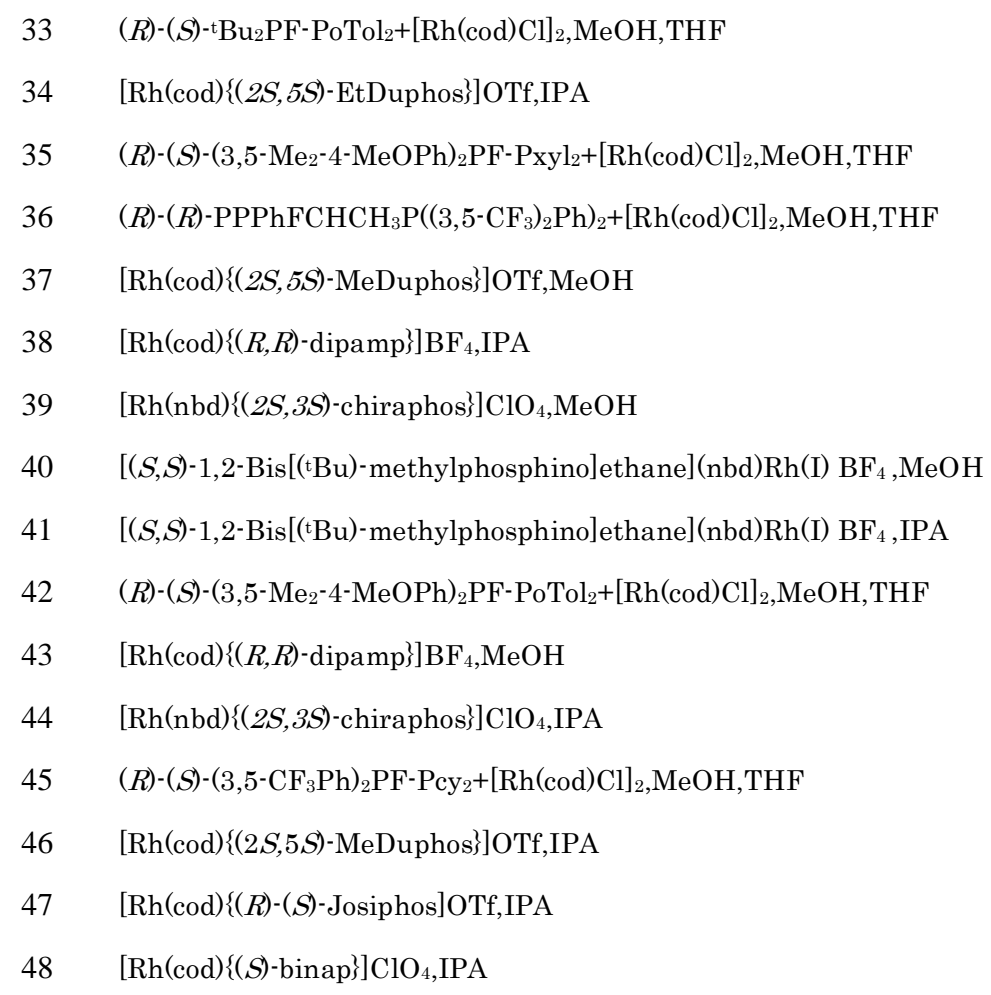

$34[\mathrm{Rh}(\operatorname{cod})\{(2 S, 5 S)$-EtDuphos $\}]$ OTf,IPA

$35(R)-(S)-\left(3,5-\mathrm{Me}_{2}-4-\mathrm{MeOPh}\right)_{2} \mathrm{PF}-\mathrm{Pxyl}_{2}+[\mathrm{Rh}(\mathrm{cod}) \mathrm{Cl}]_{2}, \mathrm{MeOH}, \mathrm{THF}$

$36 \quad(R)-(R)-\mathrm{PPPhFCHCH} \mathrm{P}_{3} \mathrm{P}\left(\left(3,5-\mathrm{CF}_{3}\right)_{2} \mathrm{Ph}\right)_{2}+\left[\mathrm{Rh}(\operatorname{cod}) \mathrm{Cl}_{2}, \mathrm{MeOH}, \mathrm{THF}\right.$

$37[\mathrm{Rh}(\operatorname{cod})\{(2 S, 5 S)-\mathrm{MeDuphos}\}] \mathrm{OTf}, \mathrm{MeOH}$

$38[\mathrm{Rh}(\operatorname{cod})\{(R, R)$-dipamp $\}] \mathrm{BF}_{4}, \mathrm{IPA}$

$39[\mathrm{Rh}(\mathrm{nbd})\{(2 S, 3 S)$-chiraphos $\}] \mathrm{ClO}_{4}, \mathrm{MeOH}$

$40 \quad\left[(S, S)\right.$-1,2-Bis $\left[\left({ }^{\mathrm{B}} \mathrm{Bu}\right)\right.$-methylphosphino]ethane](nbd) $\mathrm{Rh}(\mathrm{I}) \mathrm{BF}_{4}, \mathrm{MeOH}$

$41\left[(S, S)\right.$-1,2-Bis[(tBu)-methylphosphino] ethane](nbd)Rh(I) $\mathrm{BF}_{4}, \mathrm{IPA}$

42 (R) $-(S)-\left(3,5-\mathrm{Me}_{2}-4-\mathrm{MeOPh}\right)_{2} \mathrm{PF}-\mathrm{PoTol}_{2}+[\mathrm{Rh}(\mathrm{cod}) \mathrm{Cl}]_{2}, \mathrm{MeOH}, \mathrm{THF}$

$43[\mathrm{Rh}(\operatorname{cod})\{(R, R)$-dipamp $\}] \mathrm{BF}_{4}, \mathrm{MeOH}$

$44[\mathrm{Rh}(\mathrm{nbd})\{(2 S, 3 S)$-chiraphos $\}] \mathrm{ClO}_{4}, \mathrm{IPA}$

$45(R)-(S)-\left(3,5-\mathrm{CF}_{3} \mathrm{Ph}\right)_{2} \mathrm{PF}-\mathrm{Pcy}_{2}+[\mathrm{Rh}(\mathrm{cod}) \mathrm{Cl}]_{2}, \mathrm{MeOH}, \mathrm{THF}$

$46 \quad[\mathrm{Rh}(\operatorname{cod})\{(2 S, 5 S)-\mathrm{MeDuphos}\}] \mathrm{OTf}, \mathrm{IPA}$

$47 \quad[\operatorname{Rh}(\operatorname{cod})\{(R)-(S)$-Josiphos]OTf,IPA

$48 \quad[\mathrm{Rh}(\operatorname{cod})\{(S)$-binap $\}] \mathrm{ClO}_{4}, \mathrm{IPA}$

$\begin{array}{ccc}13.2 & 15.8 & R \\ 42.3 & 15.3 & S \\ 21.3 & 11.0 & R \\ 8.1 & 9.0 & S \\ 67.9 & 8.8 & R \\ 85.4 & 7.9 & S \\ 81.2 & 4.8 & S \\ 93.8 & 3.0 & S \\ 42.1 & 2.5 & S \\ 3.7 & 2.2 & R \\ 90.2 & 1.9 & R \\ 28.3 & 1.5 & S \\ 6.5 & 1.3 & S \\ 87.1 & 0.7 & R \\ 85.4 & 0.6 & S \\ 5.0 & 0.3 & R\end{array}$

S2-4 Ru catalyzed transfer hydrogenation (Solv. : DMF, $\mathrm{HCOOH}: \mathrm{Et}_{3} \mathrm{~N}=2.5: 1, \mathrm{~S} / \mathrm{C}=20, \mathrm{~S} / \mathrm{S}=25$, rt, $21 \mathrm{~h})$

\begin{tabular}{|c|c|c|c|c|}
\hline Entry & Catalyst & conv. & ee & config. \\
\hline 1 & $\operatorname{RuCl}\left\{(S, S)\right.$-iBuSO${ }_{2}$ dpen $\}(p$-cymene $)$ & 89.1 & 82.7 & $S$ \\
\hline 2 & $\operatorname{RuCl}\left\{(S)-\mathrm{Cs}^{-}(S, S)-\mathrm{dpen}\right\}(p$-cymene $)$ & 89.1 & 79.2 & $S$ \\
\hline 3 & $\operatorname{RuCl}\{(S, S)$-Tsdpen $\}(p$-cymene $) \mathrm{BF}_{4}$ & 96.2 & 76.5 & $S$ \\
\hline 4 & {$\left[\mathrm{Ru}(\right.$ pyridine $)(p$-cymene $)\{(S, S)-\mathrm{Tsdpen}\} \mathrm{BF}_{4}$} & 95.4 & 76.5 & $S$ \\
\hline 5 & $\operatorname{RuCl}(p$-cymene $)\{(S, S)$-Tsdpen $\}$ & 97.8 & 75.5 & $S$ \\
\hline 6 & $\operatorname{RuCl}\left\{(S, S)-\mathrm{BnSO}_{2} \mathrm{dpen}\right\}$ (p-cymene) & 86.5 & 74.9 & $S$ \\
\hline 7 & $\operatorname{RuCl}\left\{(S, S)-2^{\prime}, 6^{\prime}-\left(\mathrm{CH}_{3}\right)_{2} \mathrm{BnSO}_{2} \mathrm{dpen}\right\}(\mathrm{p}$-cymene $)$ & 61.5 & 73.3 & $S$ \\
\hline 8 & $\operatorname{RuCl}\left\{(S, S)^{-\mathrm{i}} \mathrm{BuSO}_{2} \mathrm{dpen}\right\}($ mesitylene $)$ & 65.0 & 60.9 & $S$ \\
\hline 9 & $\operatorname{RuCl}\{(S, S)$-Fsdpen $\}$ (mesitylene) & 74.4 & 60.6 & $S$ \\
\hline 10 & $\operatorname{RuCl}\{(R, R)-\mathrm{T}$ sdach $\}(p$-cymene $)$ & 26.5 & 56.6 & $R$ \\
\hline 11 & $\operatorname{RuCl}\{(S, S)$-Teth-Tsdpen $\}$ & 90.1 & 46.1 & $S$ \\
\hline 12 & $\operatorname{RuCl}\left\{(S, S)-\mathrm{BnSO}_{2} \mathrm{dpen}\right\}($ mesitylene $)$ & 66.0 & 33.2 & $S$ \\
\hline 13 & $\operatorname{RuCl}\left\{(S, S)-2^{\prime}, 6^{\prime}-\left(\mathrm{CH}_{3}\right)_{2} \mathrm{BnSO}_{2} \mathrm{dpen}\right\}($ mesitylene $)$ & 61.0 & 31.6 & $S$ \\
\hline
\end{tabular}


S2-5 Ru catalyzed transfer hydrogenation (Solv. : IPA, $\mathrm{KOH}, \mathrm{S} / \mathrm{C}=20, \mathrm{~S} / \mathrm{S}=25, \mathrm{rt}, 21 \mathrm{~h}$ )

\begin{tabular}{|c|c|c|c|c|}
\hline Entry & Catalyst & conv. & ee & config. \\
\hline 1 & $\operatorname{RuCl}\{(R, R)-\mathrm{T}$ sdach $\}(p$-cymene $)$ & 4.5 & 90.4 & $R$ \\
\hline 2 & $\operatorname{RuCl}\left\{(S, S)-2^{\prime}, 6^{\prime}-\left(\mathrm{CH}_{3}\right)_{2} \mathrm{BnSO}_{2} \mathrm{dpen}\right\}$ (mesitylene) & 1.5 & 68.5 & $R$ \\
\hline 3 & $\operatorname{RuCl}\left\{(S)-\mathrm{Cs}^{-}(S, S)-\operatorname{dpen}\right\}(p$-cymene $)$ & 1.9 & 64.7 & $S$ \\
\hline 4 & $\operatorname{RuCl}(p$-cymene $)\{(S, S)$-Tsdpen $\}$ & 1.3 & 64.7 & - \\
\hline 5 & $\operatorname{RuCl}\left\{(S, S)-\mathrm{BnSO}_{2} \mathrm{dpen}\right\}($ mesitylene $)$ & 2.0 & 52.3 & $R$ \\
\hline 6 & $\operatorname{RuCl}\left\{(S, S)-2^{\prime}, 6^{\prime}-\left(\mathrm{CH}_{3}\right)_{2} \mathrm{BnSO}_{2} \mathrm{dpen}\right\}(\mathrm{p}$-cymene $)$ & 4.6 & 35.8 & $S$ \\
\hline 7 & $\operatorname{RuCl}\{(S, S)$-Teth-Tsdpen $\}$ & 5.8 & 27.1 & $R$ \\
\hline 8 & $\operatorname{RuCl}\left\{(S, S)\right.$-i $\mathrm{BuSO}_{2}$ dpen $\}$ (mesitylene) & 0.8 & 8.9 & $S$ \\
\hline 9 & $\operatorname{RuCl}\left\{(S, S){ }^{-1} \mathrm{BuSO}_{2} \mathrm{dpen}\right\}(p$-cymene $)$ & 4.2 & 7.2 & $R$ \\
\hline 10 & {$[\mathrm{Ru}($ pyridine $)(p$-cymene $)\{(S, S)-\mathrm{Tsdpen}\}] \mathrm{BF}_{4}$} & 1.5 & 5.9 & - \\
\hline 11 & $\operatorname{RuCl}\{(S, S)-F s d p e n\}($ mesitylene $)$ & 0.8 & 5.2 & $S$ \\
\hline 12 & $\operatorname{RuCl}\{(S, S)$-Tsdpen $\}(p$-cymene $) \mathrm{BF}_{4}$ & 1.3 & 0.0 & - \\
\hline 13 & $\operatorname{RuCl}\left\{(S, S)-\mathrm{BnSO}_{2} \mathrm{dpen}\right\}(\mathrm{p}$-cymene $)$ & 0.0 & 0.0 & - \\
\hline
\end{tabular}


Table S3 Screening of conditions for Cu mediated $N$-arylation.

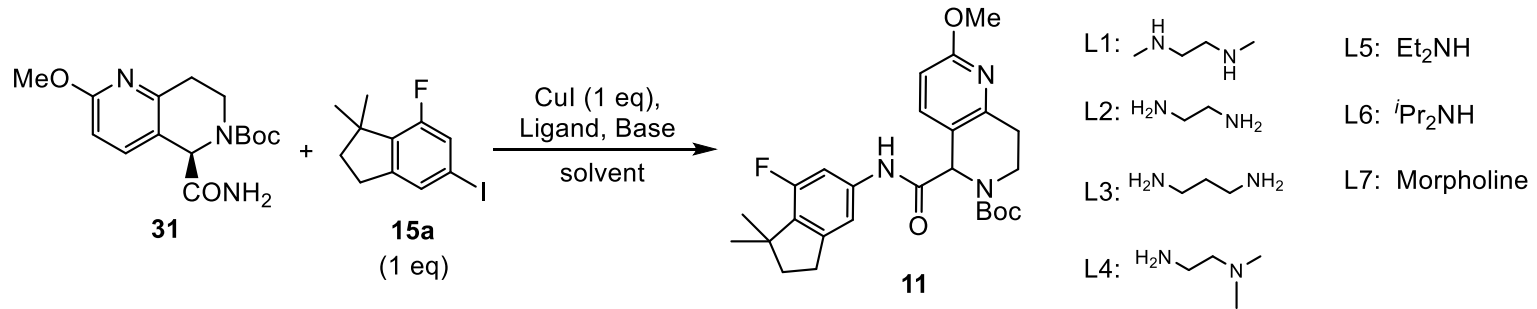

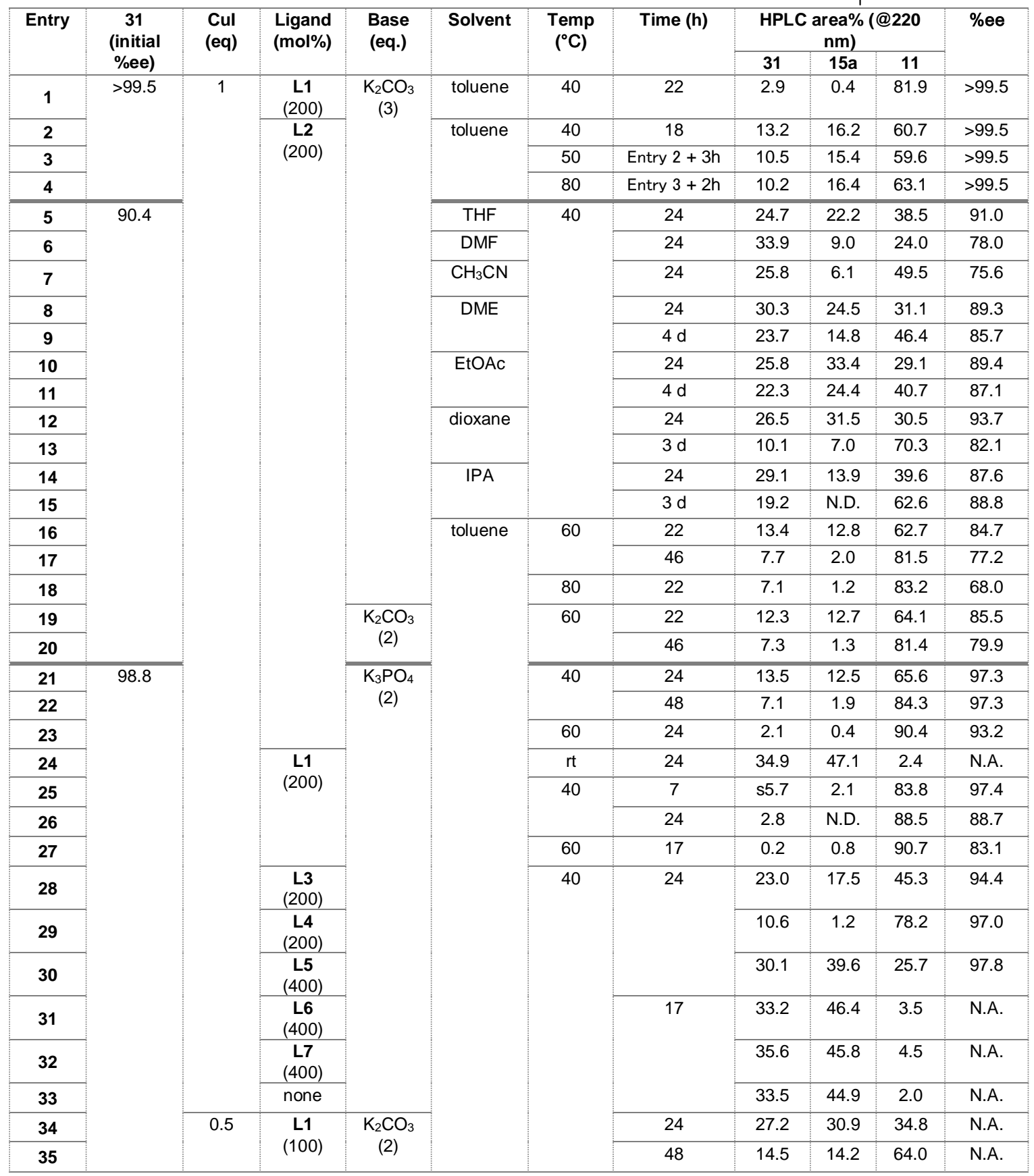

N.D. $=$ Not detected. N.A. $=$ Not analyzed. 
$21\left({ }^{1} \mathrm{H}\right.$ NMR, $\left.\mathrm{CDCl}_{3}, 500 \mathrm{MHz}\right)$

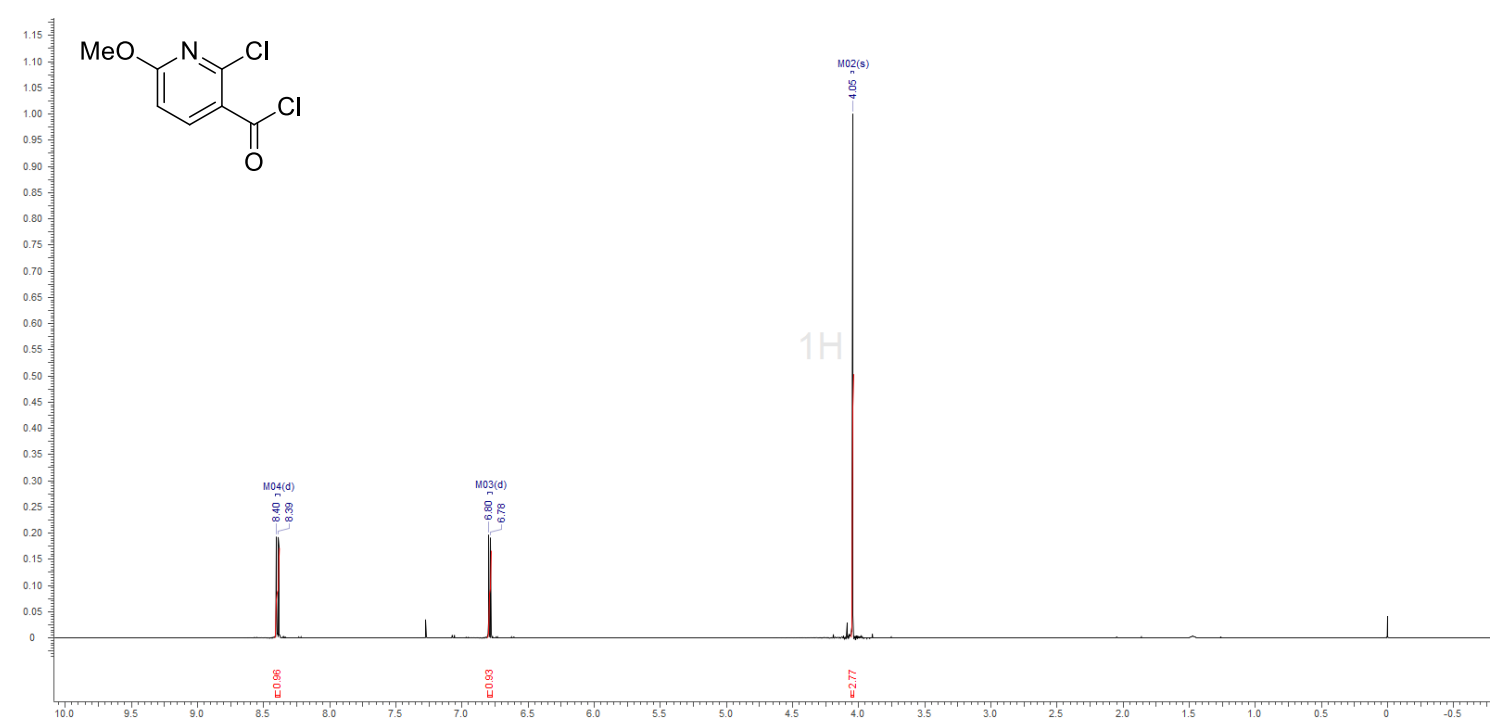

$\left({ }^{13} \mathrm{C} \mathrm{NMR}, \mathrm{CDCl}_{3}, 126 \mathrm{MHz}\right)$

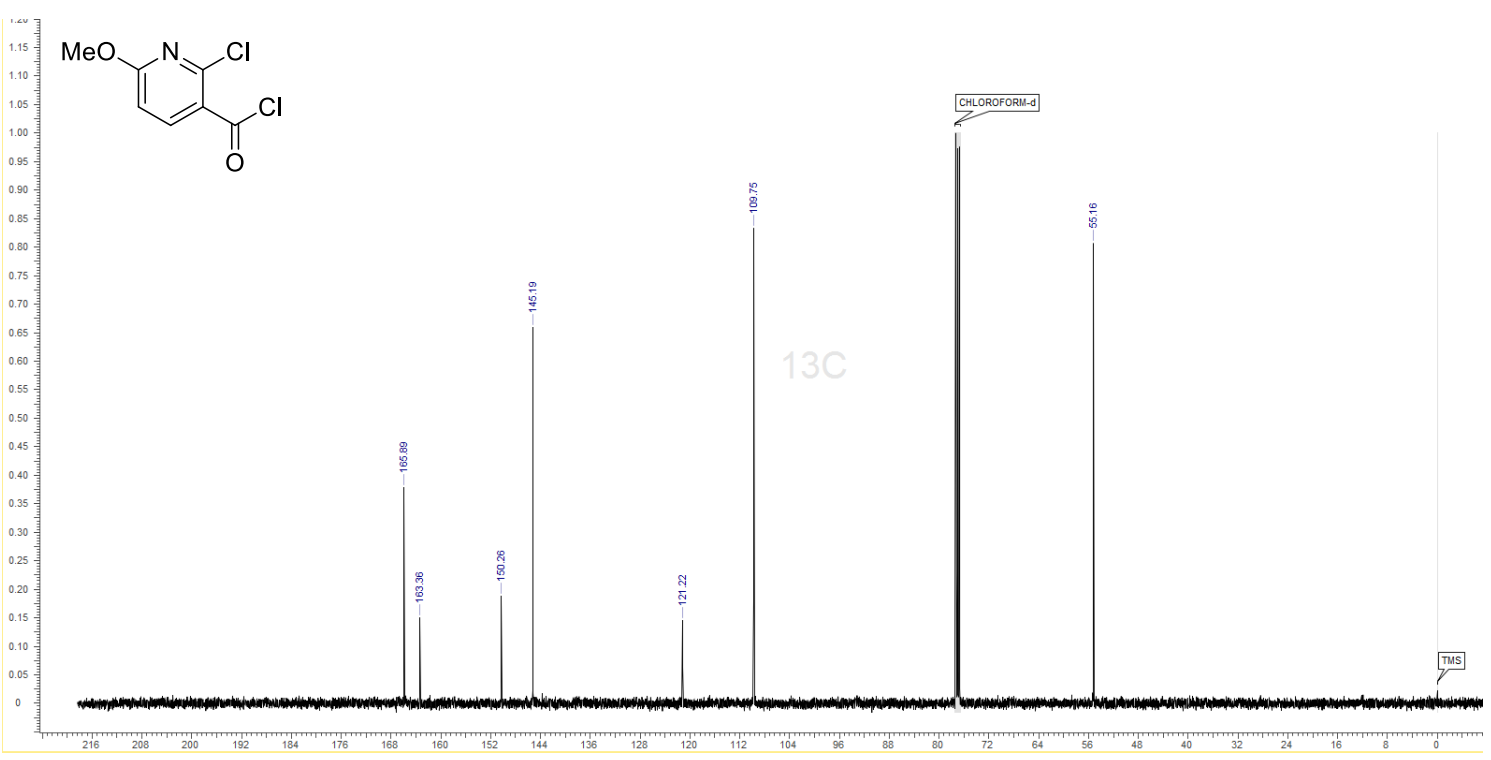


$22\left({ }^{1} \mathrm{H} \mathrm{NMR}, \mathrm{CDCl}_{3}, 500 \mathrm{MHz}\right)$

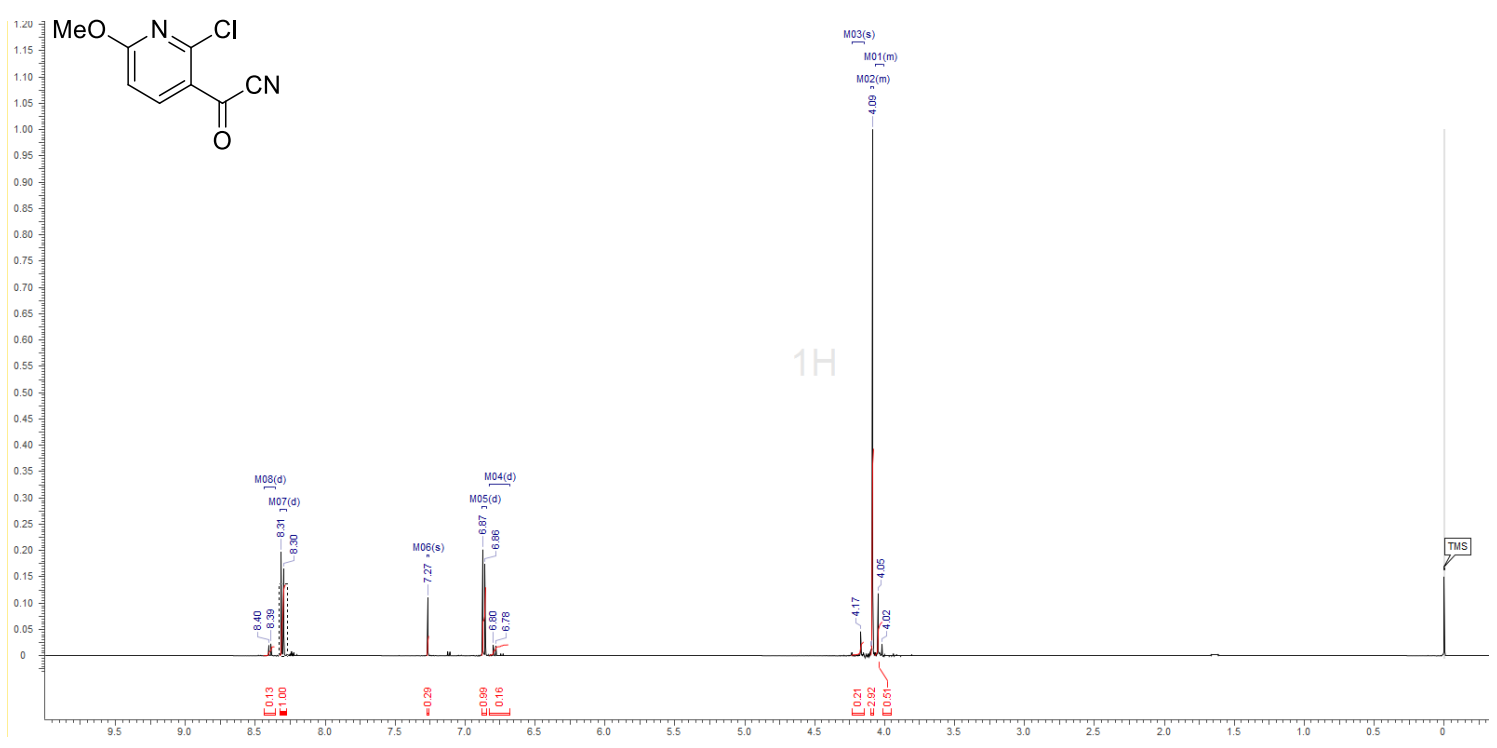

$\left({ }^{13} \mathrm{C} \mathrm{NMR}, \mathrm{CDCl}_{3}, 126 \mathrm{MHz}\right)$

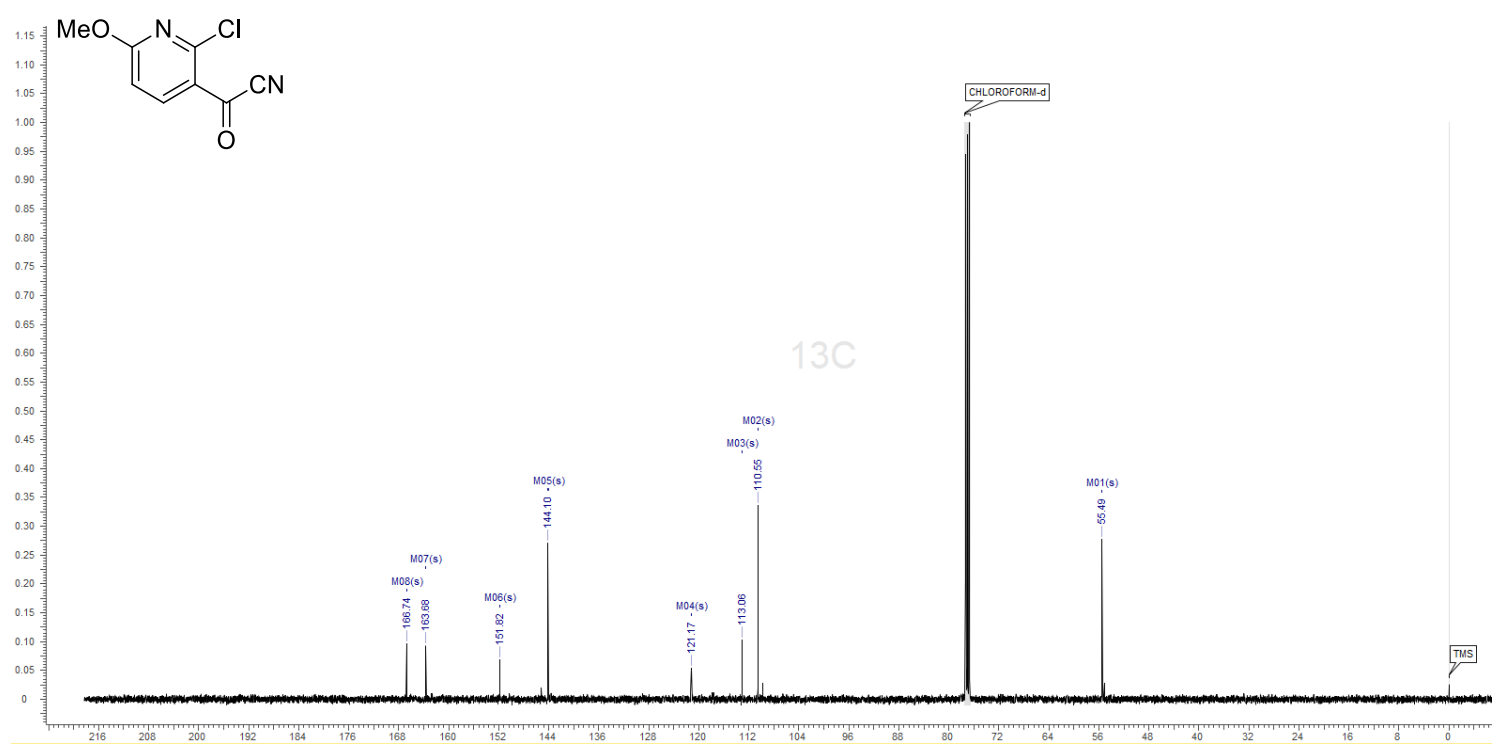


$25\left({ }^{1} \mathrm{H}\right.$ NMR, $\left.\mathrm{CDCl}_{3}, 500 \mathrm{MHz}\right)$

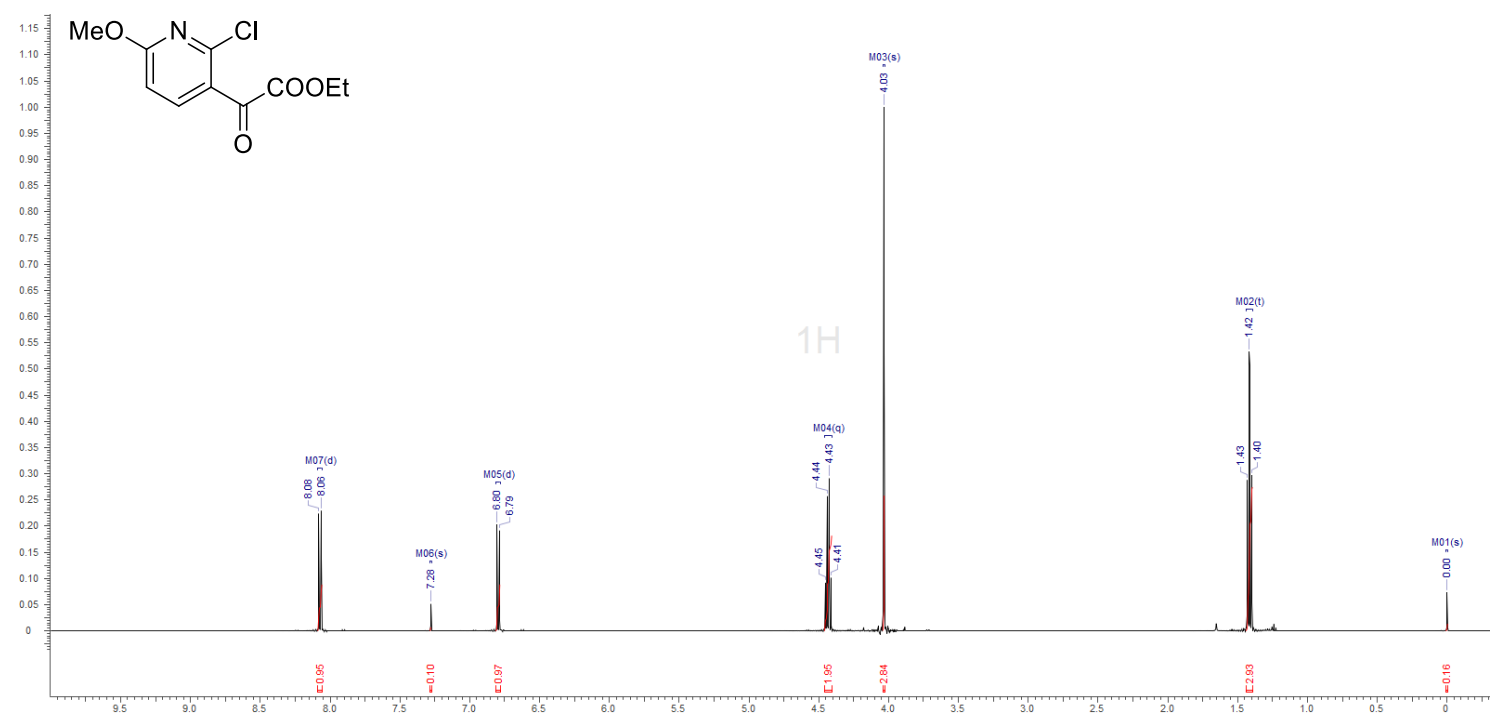

$\left({ }^{13} \mathrm{C} \mathrm{NMR}, \mathrm{CDCl}_{3}, 126 \mathrm{MHz}\right)$

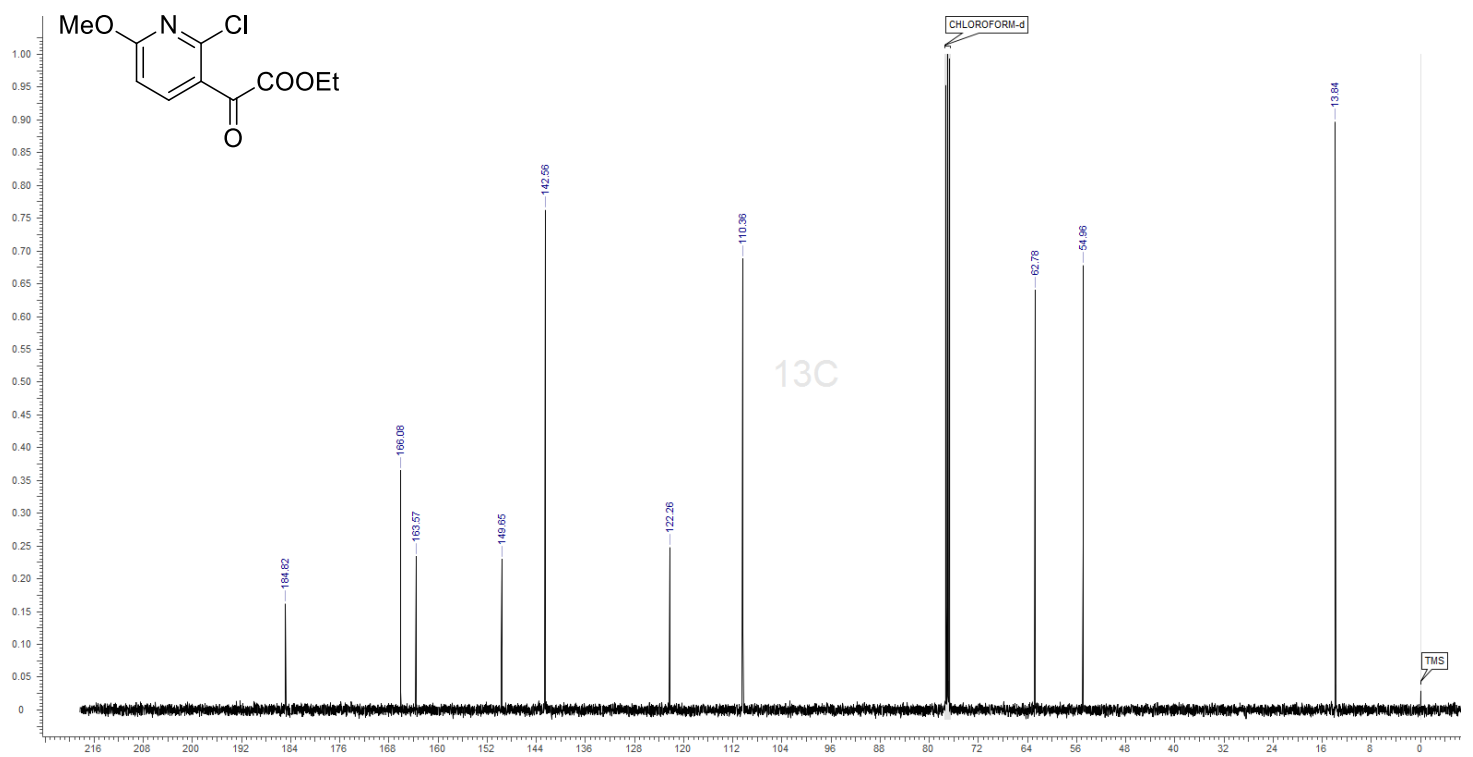


23 ( ${ }^{1} \mathrm{H}$ NMR, DMSO- $\left.d_{6}, 500 \mathrm{MHz}\right)$

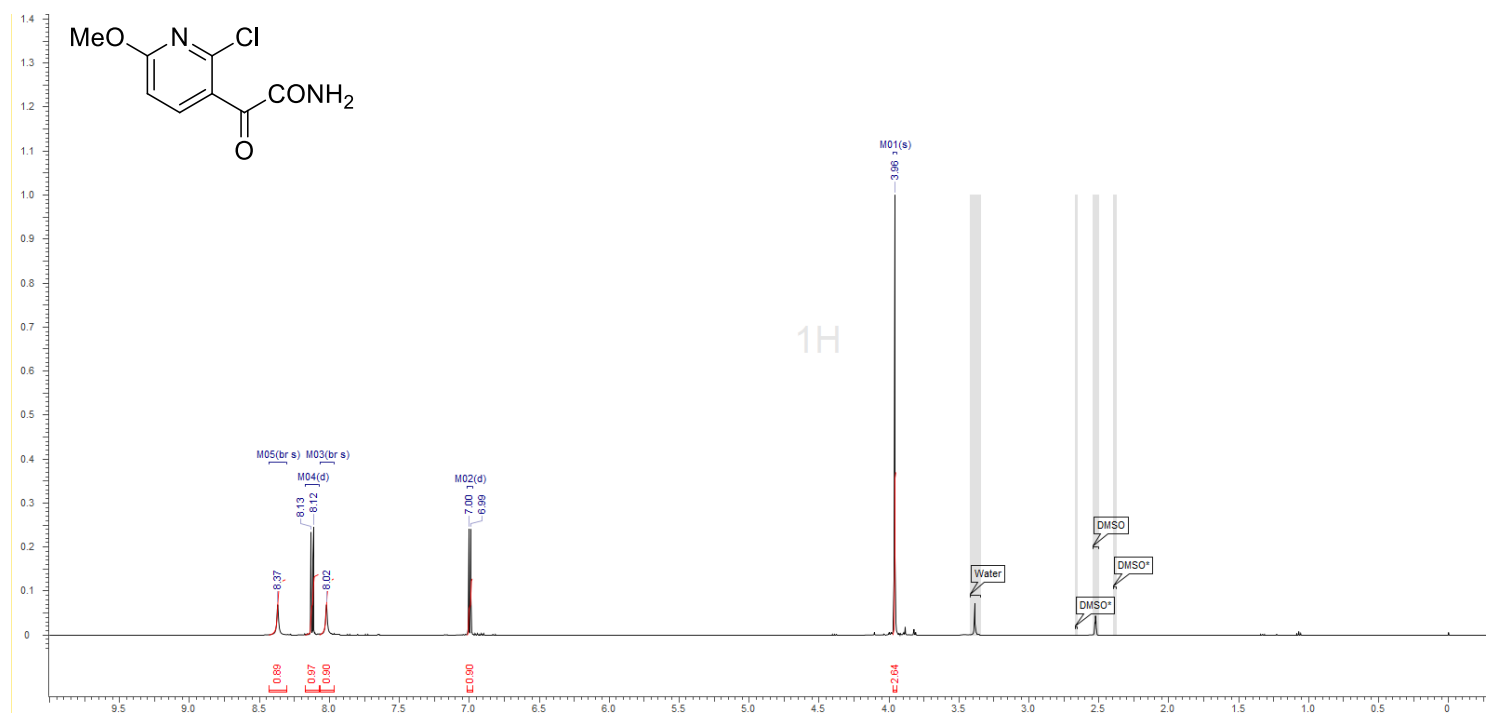

$\left({ }^{13} \mathrm{C}\right.$ NMR, DMSO- $\left.d_{6}, 126 \mathrm{MHz}\right)$

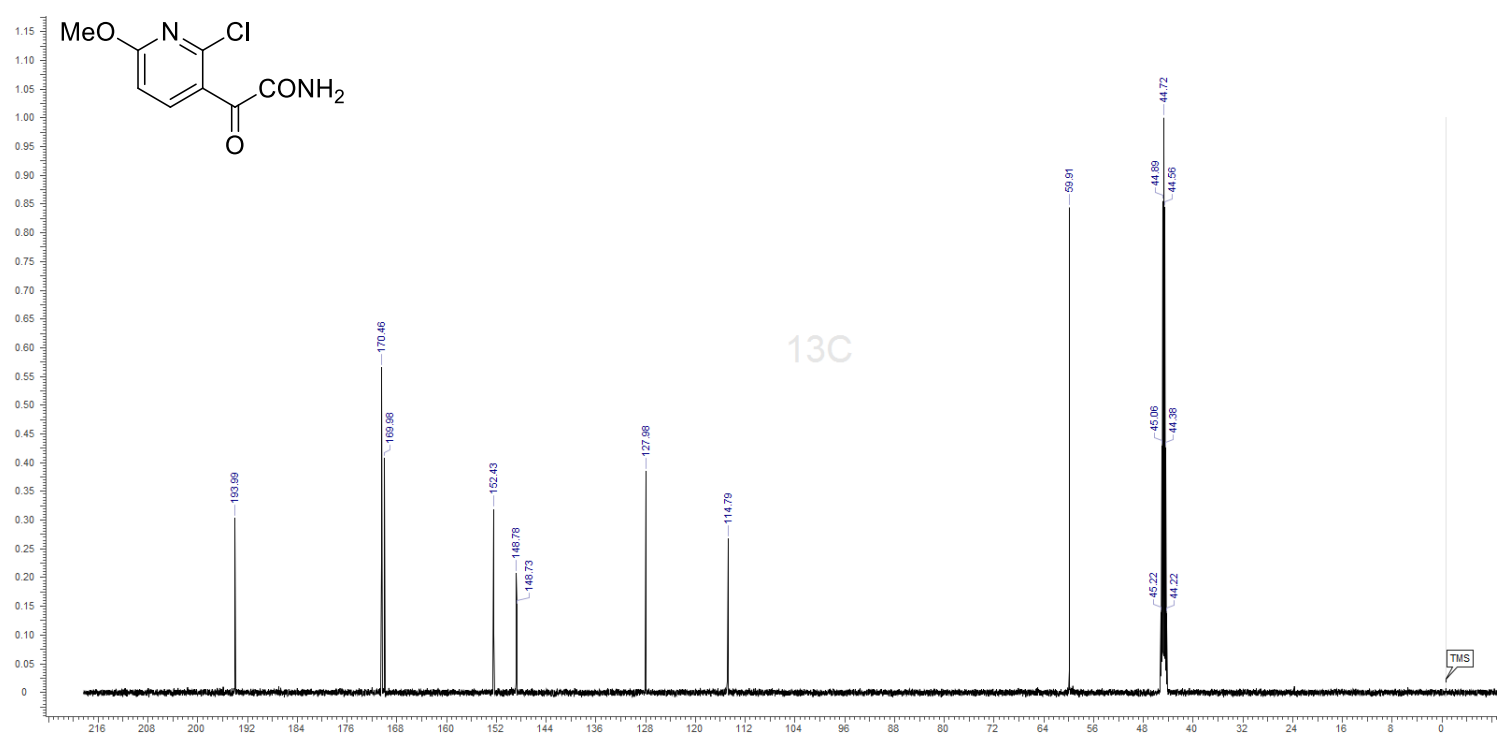


$19\left({ }^{1} \mathrm{H}\right.$ NMR, DMSO- $\left.d_{6}, 500 \mathrm{MHz}\right)$

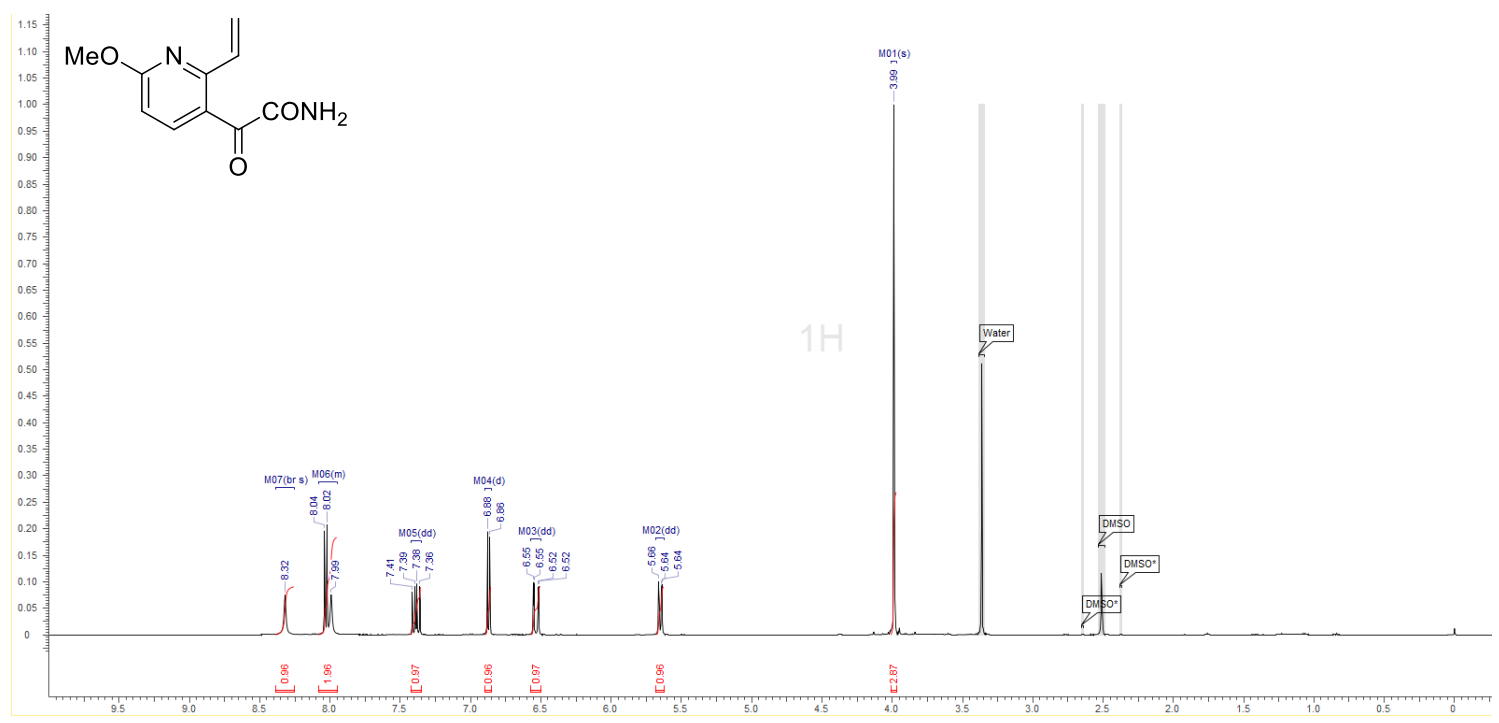

$\left({ }^{13} \mathrm{C}\right.$ NMR, DMSO- $\left.d_{6}, 126 \mathrm{MHz}\right)$

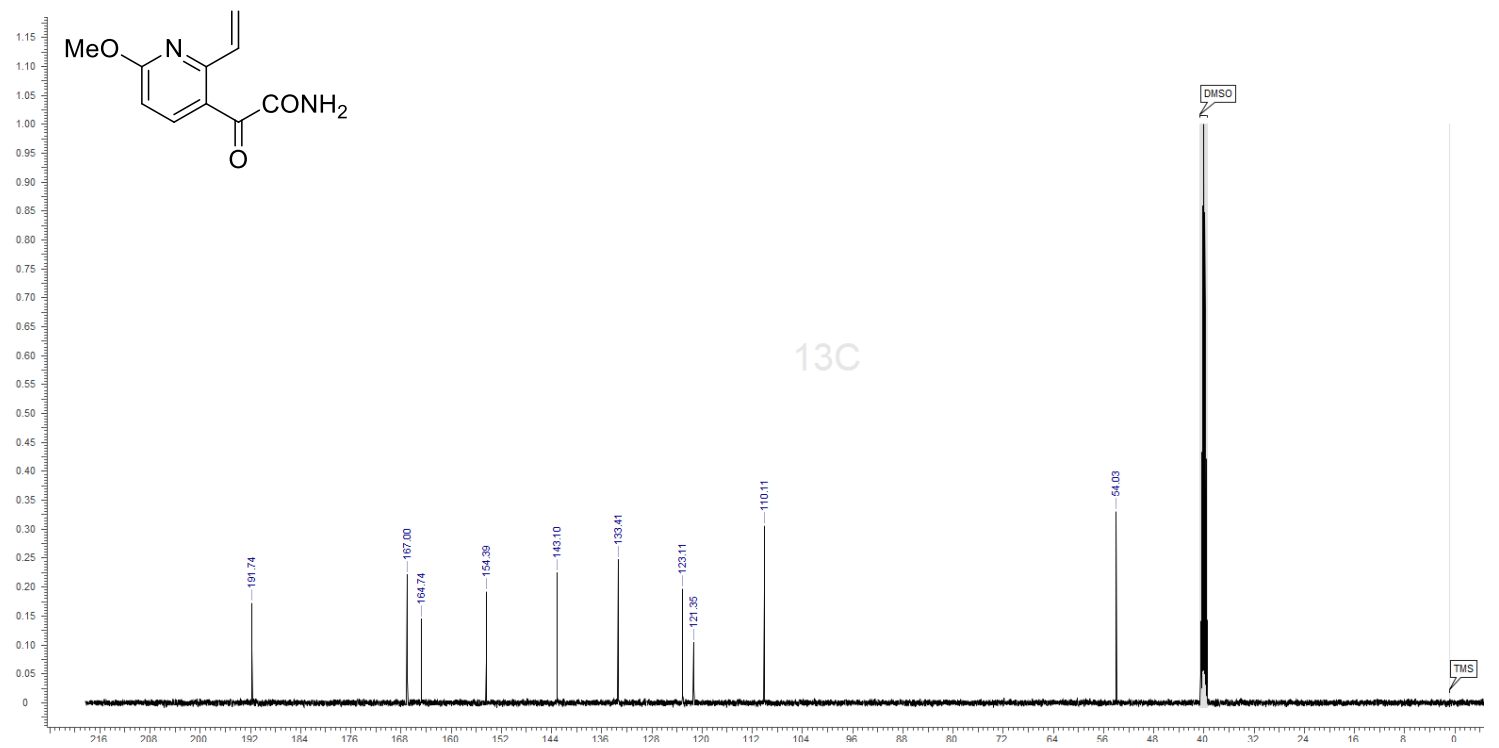


$17\left({ }^{1} \mathrm{H}\right.$ NMR, DMSO- $\left.d_{6}, 500 \mathrm{MHz}\right)$

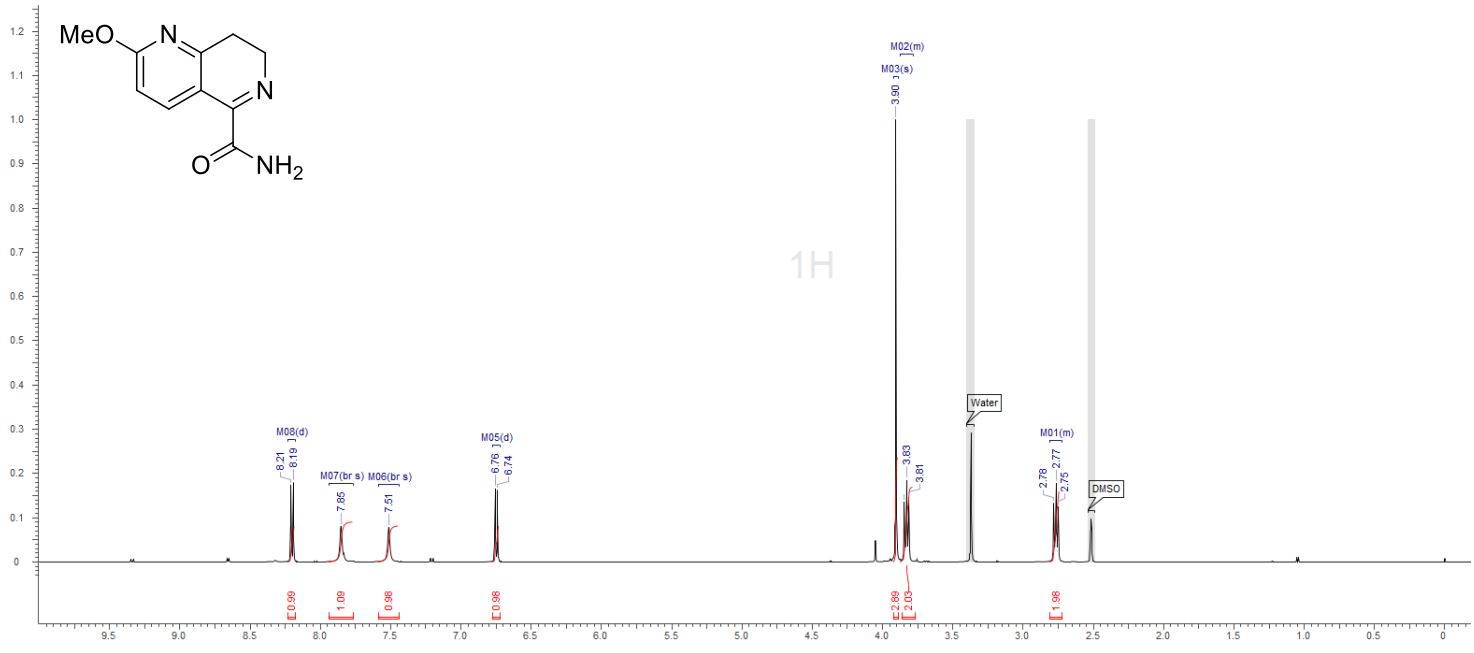

$\left({ }^{13} \mathrm{C}\right.$ NMR, DMSO- $\left.d_{6}, 126 \mathrm{MHz}\right)$

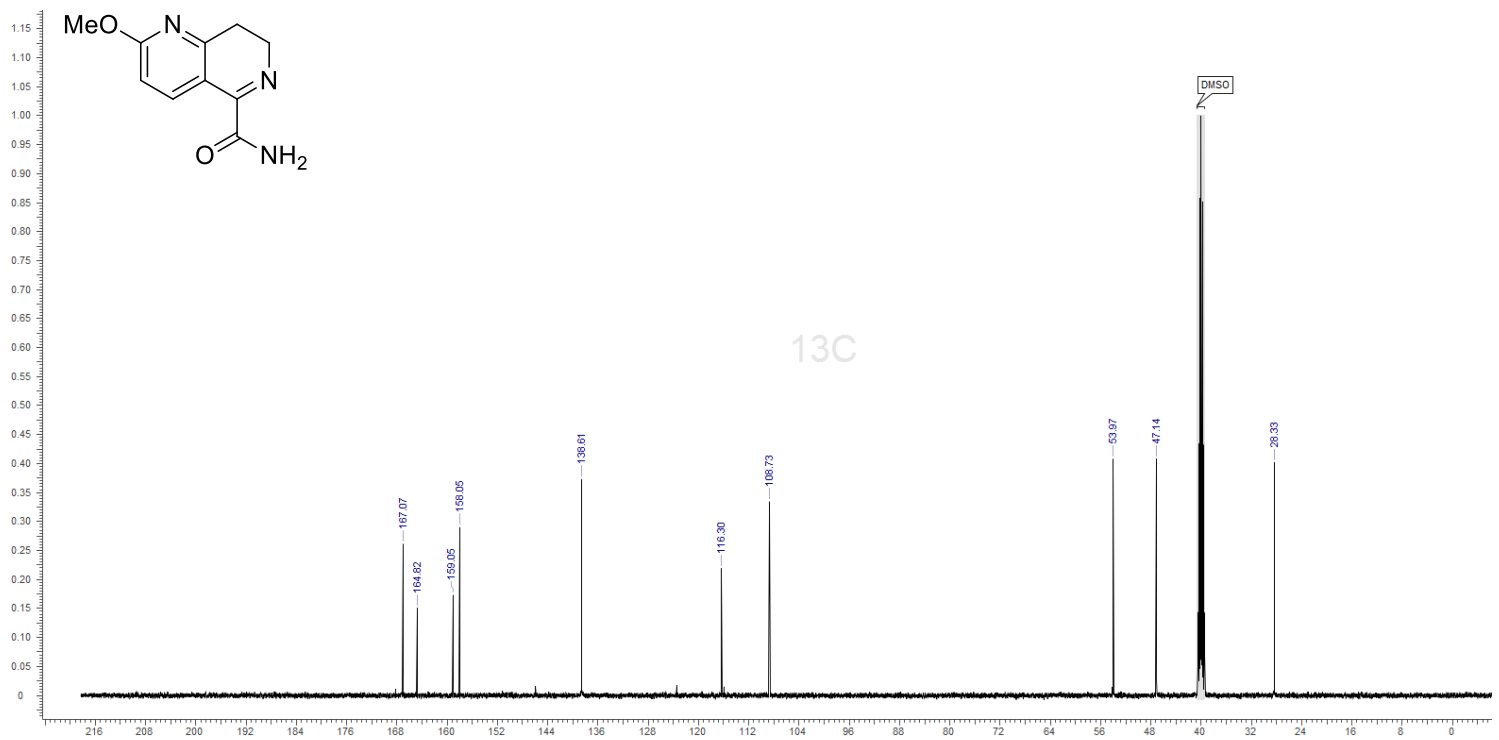


$31\left({ }^{1} \mathrm{H}\right.$ NMR, DMSO- $\left.d_{6}, 500 \mathrm{MHz}\right)$

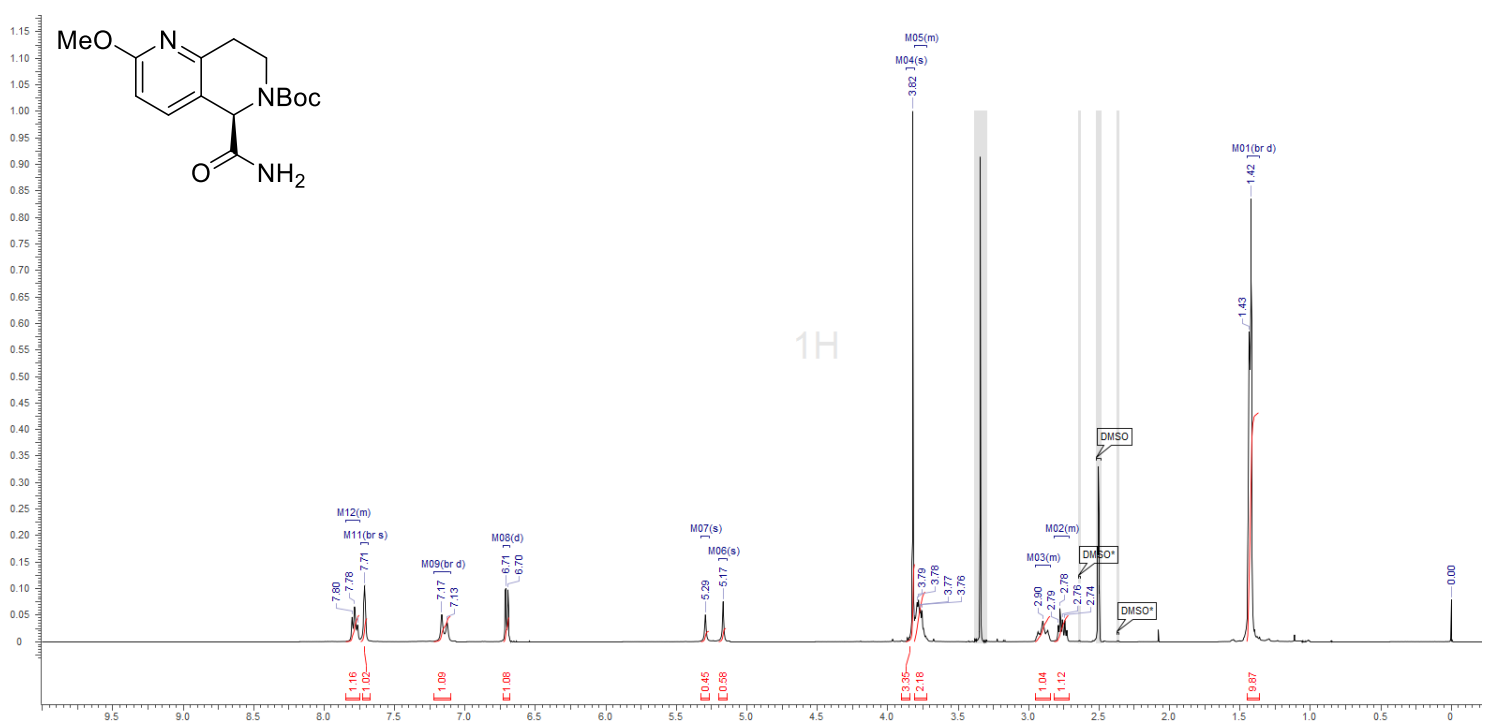

$\left({ }^{13} \mathrm{C}\right.$ NMR, DMSO- $\left.d_{6}, 126 \mathrm{MHz}\right)$

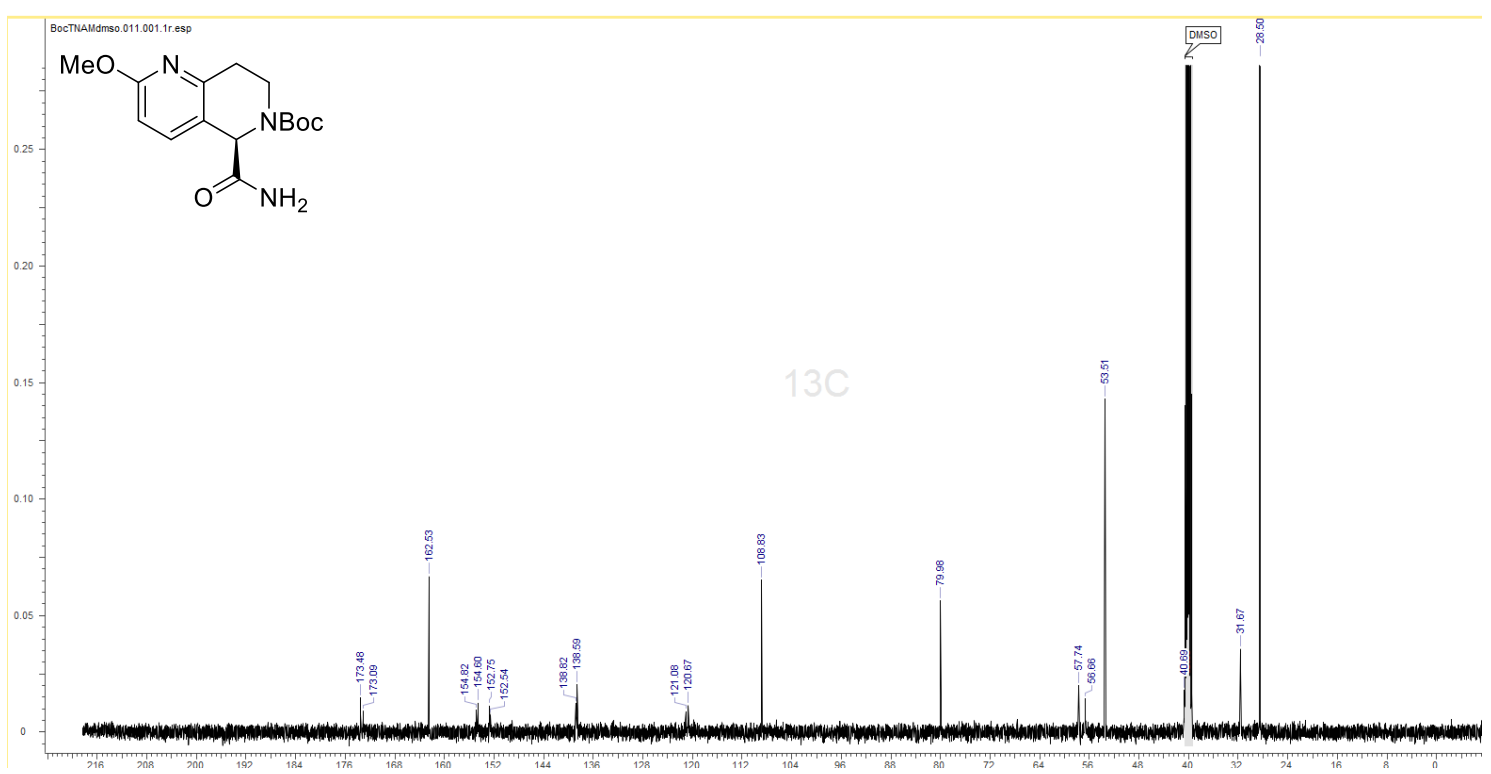

Chiral HPLC chart (black line: $(R)-\mathbf{3 1}$, blue line: racemic-31):

Column: DAICEL CHIRALCEL OZ-3R (4.6 mm*150 mm); Flow rate: $1 \mathrm{~mL} / \mathrm{min}$; Column temp: 30

${ }^{\circ} \mathrm{C}$; Eluent: $\mathrm{CH}_{3} \mathrm{CN}: 25 \mathrm{mM}$ aq. $\mathrm{AcONH}_{4}=2: 8$; Detector: UV $220 \mathrm{~nm}$

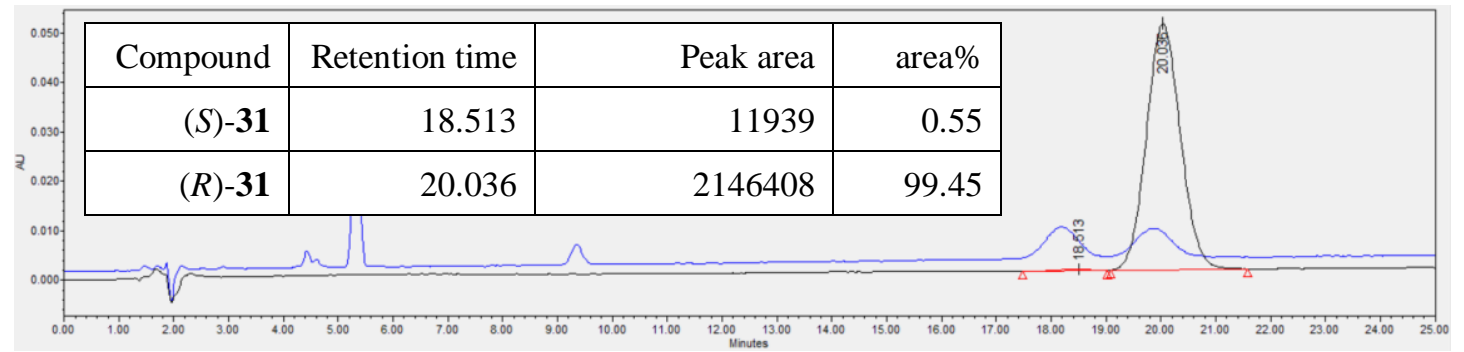


$16\left({ }^{1} \mathrm{H}\right.$ NMR, DMSO- $\left.d_{6}, 500 \mathrm{MHz}\right)$

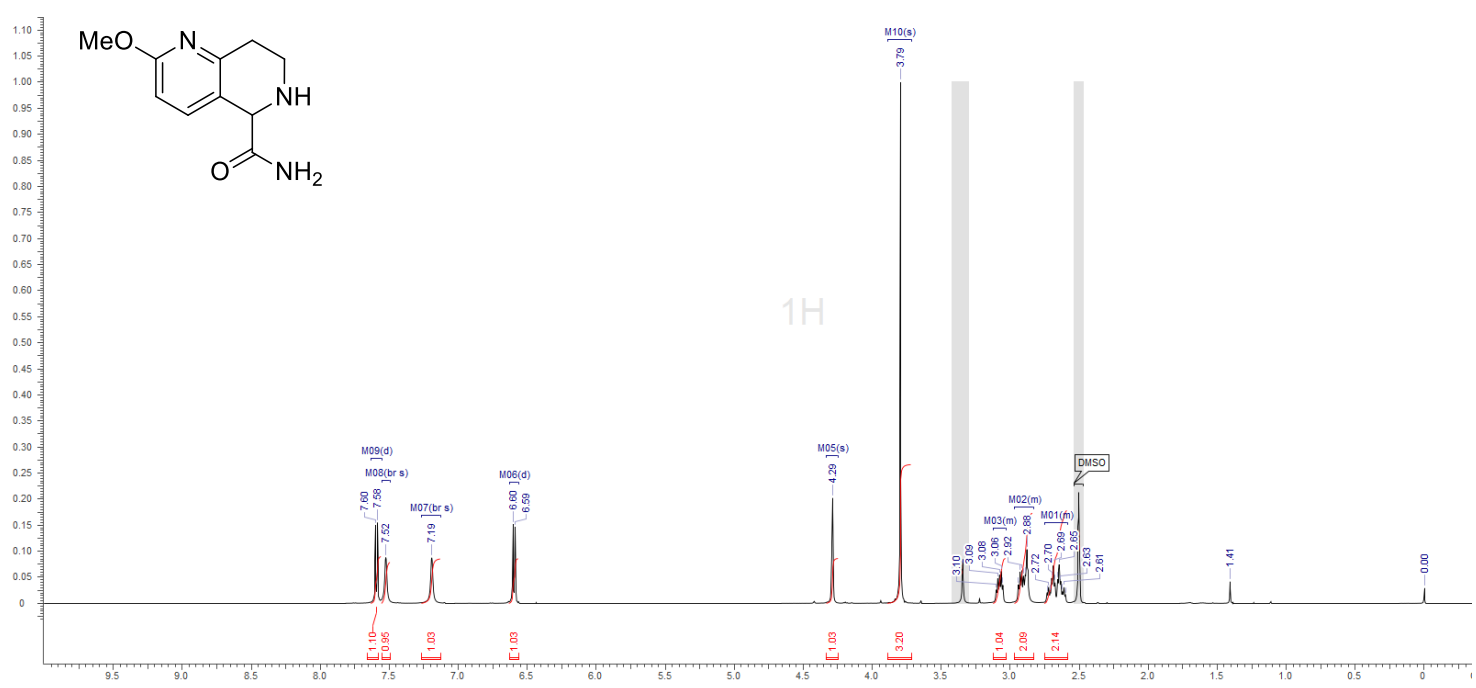

$\left({ }^{13} \mathrm{C}\right.$ NMR, DMSO- $\left.d_{6}, 126 \mathrm{MHz}\right)$

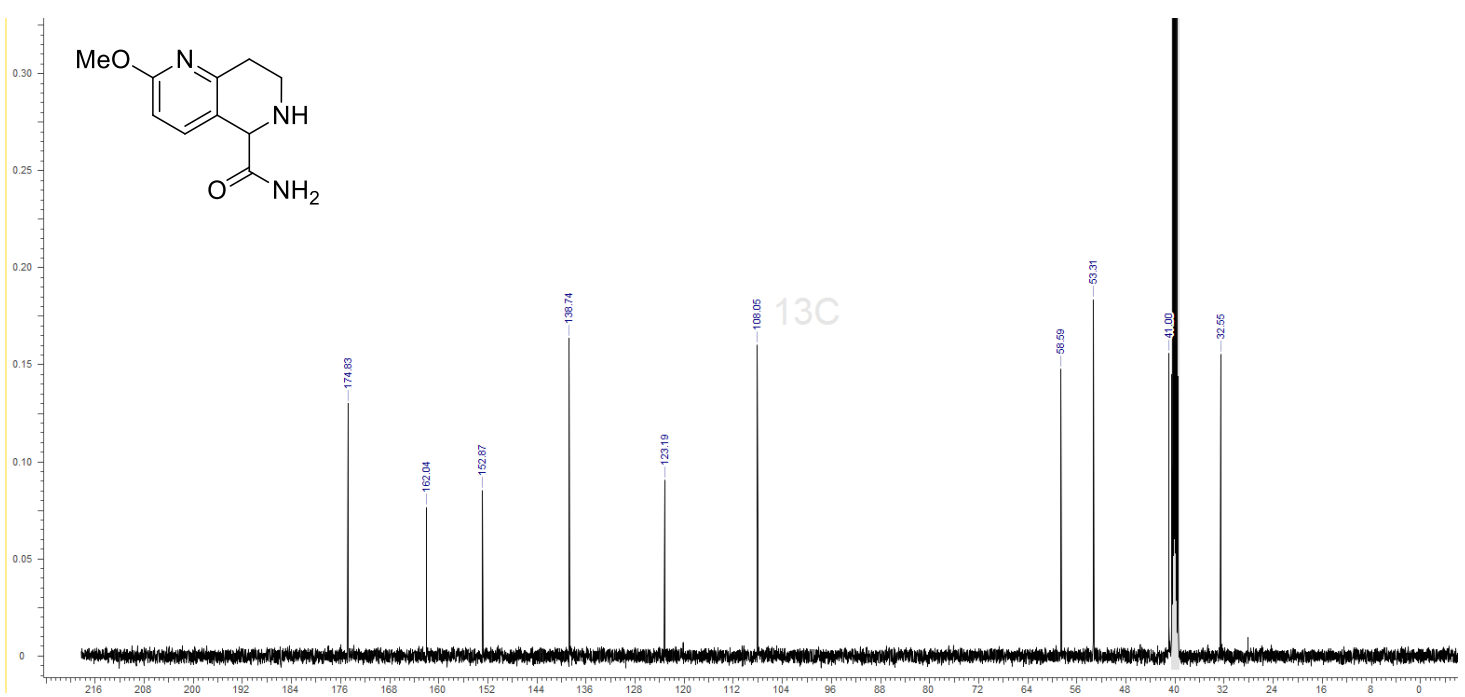


$11\left({ }^{1} \mathrm{H} \mathrm{NMR}, \mathrm{CDCl}_{3}, 500 \mathrm{MHz}\right)$

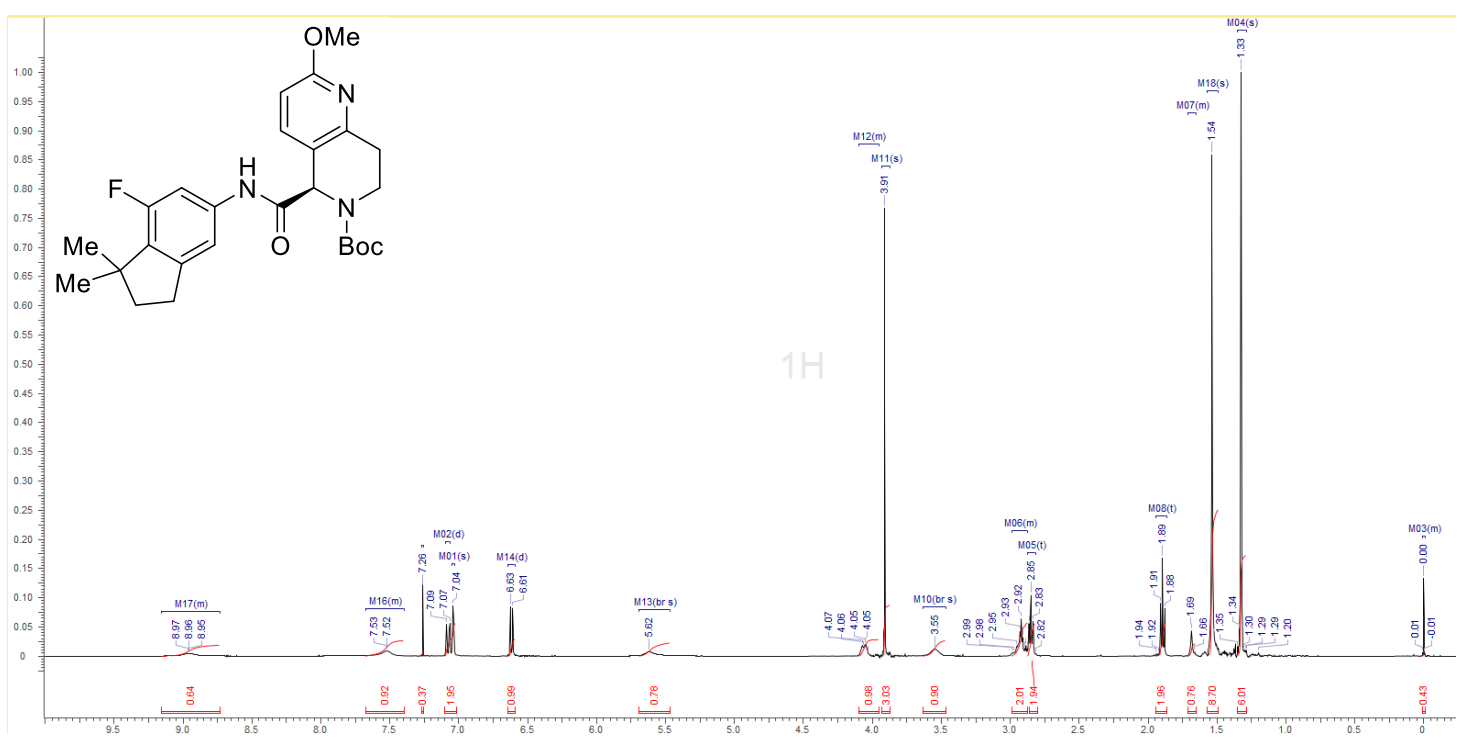

$\left({ }^{13} \mathrm{C} \mathrm{NMR}, \mathrm{CDCl}_{3}, 126 \mathrm{MHz}\right)$

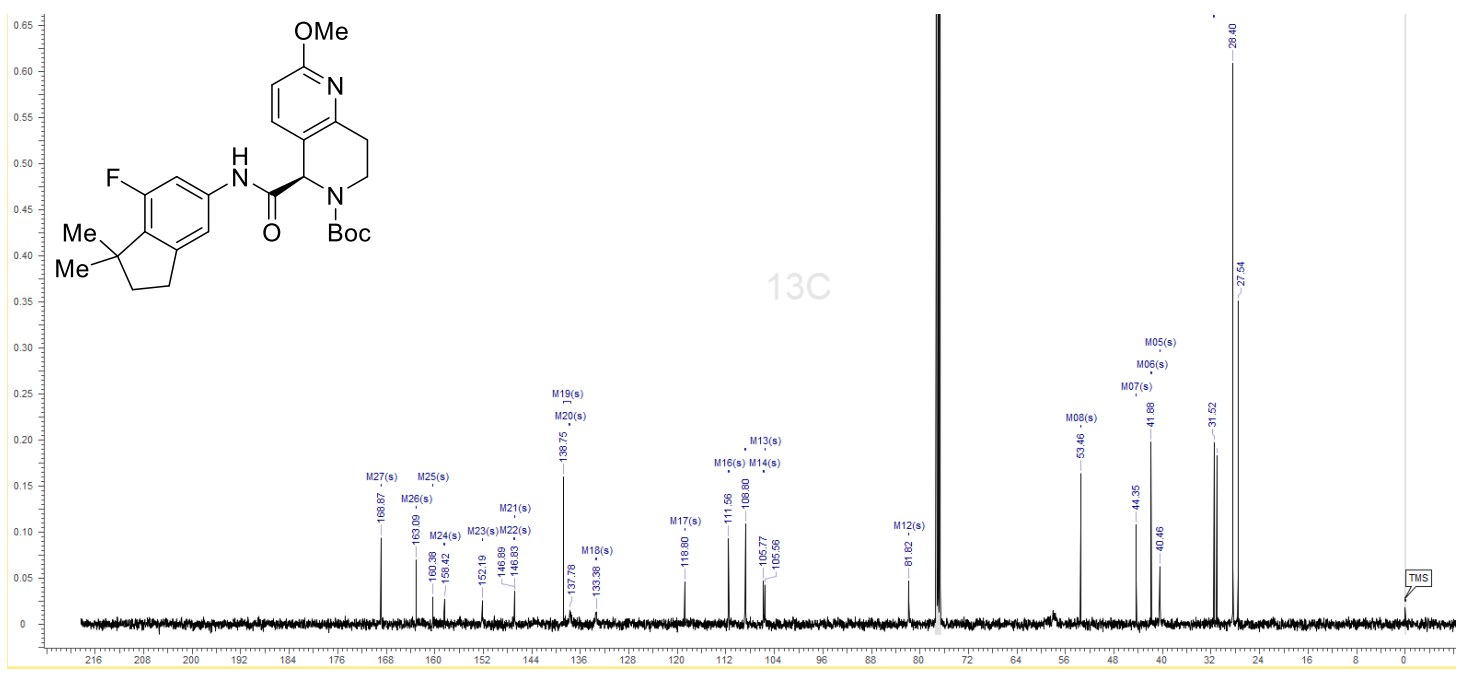

Chiral HPLC chart (black line: $(R)$-11, blue line: $(R, S)$-mixture of 11):

Column: DAICEL CHIRALCEL OZ-3R $(4.6 \mathrm{~mm} * 150 \mathrm{~mm})$; Flow rate: $1 \mathrm{~mL} / \mathrm{min}$; Column temp: 30 ${ }^{\circ} \mathrm{C}$; Eluent: $\mathrm{CH}_{3} \mathrm{CN}: 0.1 \%$ aq. $\mathrm{H}_{3} \mathrm{PO}_{4}=4: 6$; Detector: UV $220 \mathrm{~nm}$

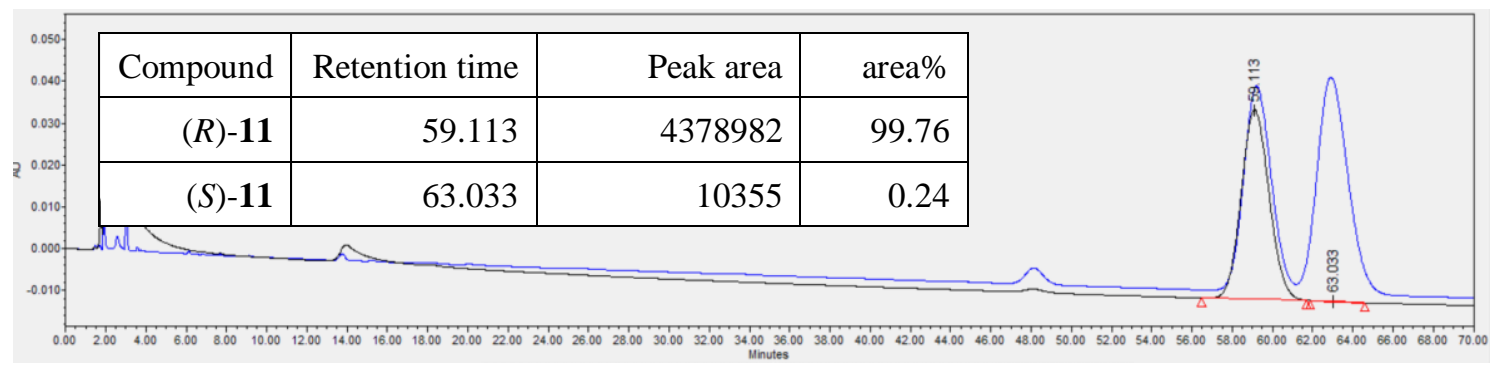


15a ( $\left.{ }^{1} \mathrm{H} \mathrm{NMR}, \mathrm{CDCl}_{3}, 500 \mathrm{MHz}\right)$

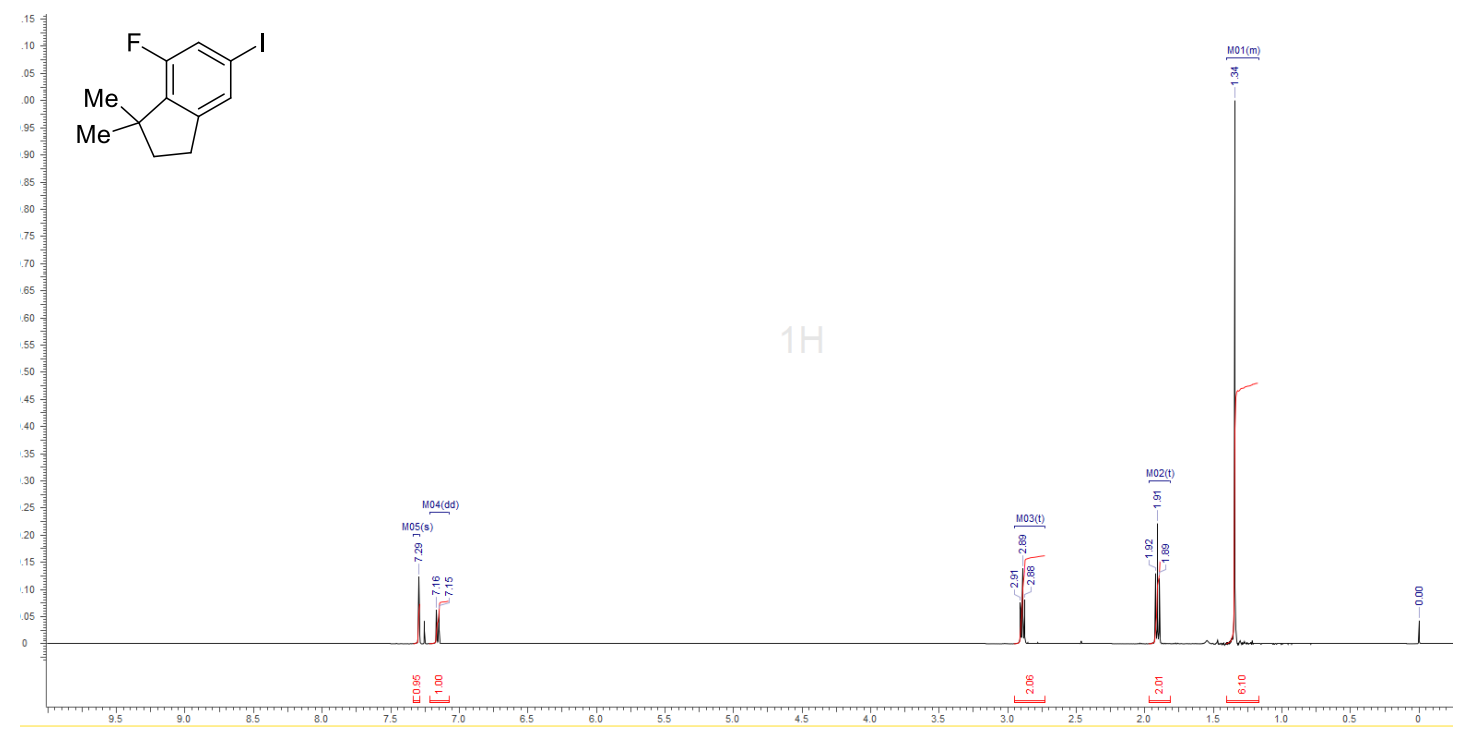

$\left({ }^{13} \mathrm{C}\right.$ NMR, $\left.\mathrm{CDCl}_{3}, 126 \mathrm{MHz}\right)$

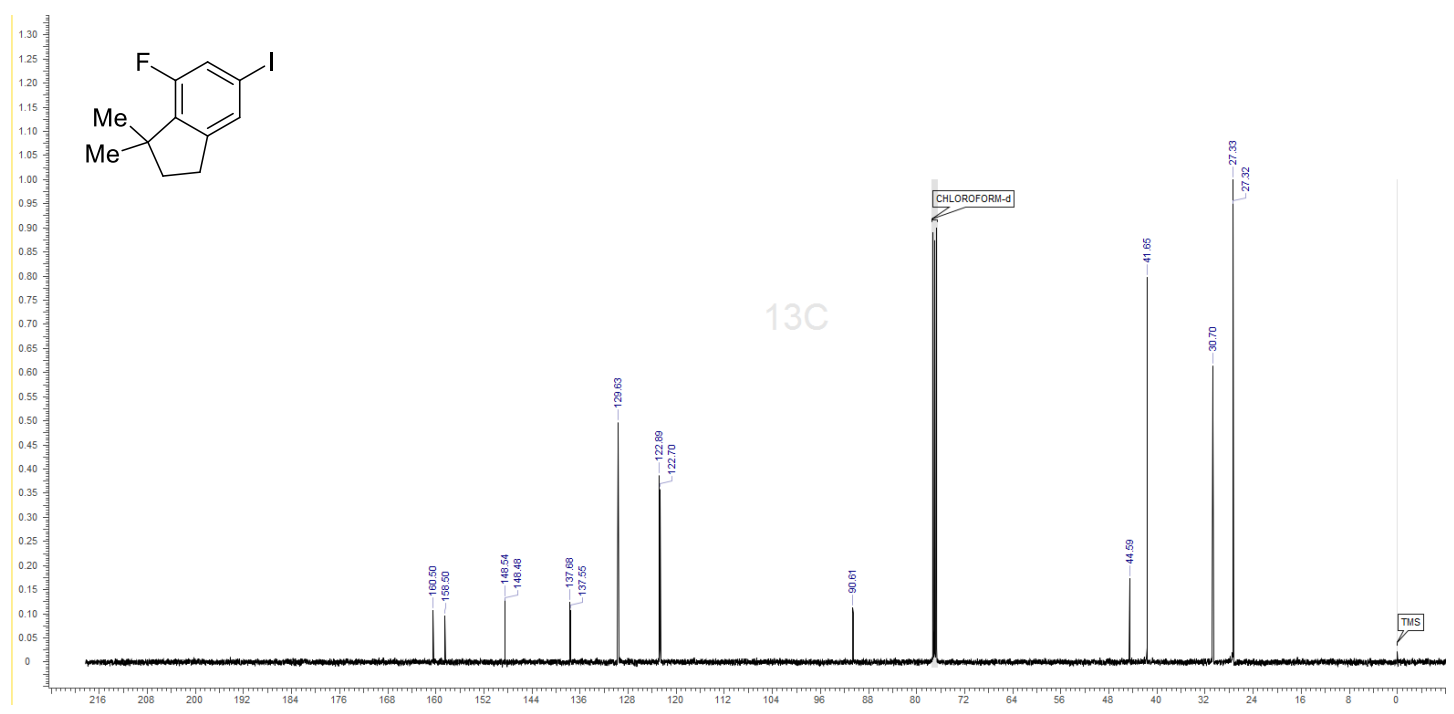

